\title{
openheart Efficacy of cilostazol on platelet reactivity and cardiovascular outcomes in patients undergoing percutaneous coronary intervention: insights from a meta-analysis of randomised trials
}

\author{
Sripal Bangalore, ${ }^{1}$ Amita Singh, ${ }^{1}$ Bora Toklu, ${ }^{1}$ James J DiNicolantonio, ${ }^{2}$ \\ Kevin Croce, ${ }^{3}$ Frederick Feit, ${ }^{1}$ Deepak L Bhatt ${ }^{3,4}$
}

To cite: Bangalore $\mathrm{S}$, Singh A, Toklu B, et al. Efficacy of cilostazol on platelet reactivity and cardiovascular outcomes in patients undergoing percutaneous coronary intervention: insights from a meta-analysis of randomised trials. Open Heart 2014;1:e000068.

doi:10.1136/openhrt-2014000068

- Additional material is available. To view please visit the journal online (http://dx. doi.org/10.1136/openhrt2014-000068).

Received 19 February 2014 Revised 30 May 2014 Accepted 15 July 2014

CrossMark

For numbered affiliations see end of article.

Correspondence to Dr Sripal Bangalore; sripalbangalore@gmail.com

\section{ABSTRACT}

Background: Cilostazol overcomes high on-treatment platelet reactivity (HTPR) and reduces adverse cardiovascular (CV) outcomes after percutaneous coronary intervention $(\mathrm{PCl})$. However, the role for triple antiplatelet therapy (TAPT) with cilostazol in addition to aspirin and clopidogrel after $\mathrm{PCl}$ is not well defined.

Methods: We conducted a MEDLINE/EMBASE/

CENTRAL search for randomised trials, until May 2014, evaluating TAPT compared with dual antiplatelet therapy (DAPT) of aspirin and clopidogrel alone in patients undergoing $\mathrm{PCl}$ and reporting platelet reactivity and/or CV outcomes. The primary platelet reactivity outcome was differences in platelet reactivity unit (PRU) with secondary outcomes of \%platelet inhibition and rate of HTPR. The primary CV outcome was major adverse cardiovascular events (MACE), with secondary outcomes of death, cardiovascular death, myocardial infarction, stent thrombosis (ST), target lesion revascularisation (TLR) and target vessel revascularisation (TVR) as well as safety outcomes of bleeding and drug discontinuations.

Results: In 17 trials that evaluated platelet reactivity outcomes, the mean PRU value was 47.73 units lower with TAPT versus DAPT $(95 \% \mathrm{Cl}-61.41$ to -34.04 , $\mathrm{p}<0.0001$; mean PRU 182.90 vs 232.65 ). TAPT also increased platelet inhibition by $12.71 \%(95 \% \mathrm{Cl} 10.76$ to $14.67, p<0.0001$ ), and led to a $60 \%$ reduction in the risk of HTPR (relative risk $=0.40 ; 95 \% \mathrm{Cl} 0.30$ to 0.53 ) compared with DAPT. Moreover, among the 34 trials that evaluated CV outcomes, TAPT reduced the risk of MACE (incident rate ratio (IRR) $=0.68 ; 95 \% \mathrm{Cl} 0.60$ to 0.78), TLR (IRR=0.57; $95 \% \mathrm{Cl} 0.44$ to 0.73 ), TVR (IRR=0.69; $95 \% \mathrm{Cl} 0.59$ to 0.81 ) and ST (IRR=0.63; $95 \% \mathrm{Cl} 0.40$ to 0.98 ) with no difference for other outcomes including bleeding, even in trials using drugeluting stents. Drug discontinuation due to adverse effects was, however, higher with TAPT vs DAPT (IRR=1.59; $95 \% \mathrm{Cl} 1.32$ to 1.91 ).

Conclusions: In patients undergoing $\mathrm{PCl}$, addition of cilostazol to DAPT results in decreased platelet reactivity and a significant reduction in CV outcomes including ST, even in the drug-eluting stent era.

\section{KEY MESSAGES}

What is already known about this subject?

- Cilostazol, a phosphodiesterase III inhibitor, exhibits antiplatelet effect and inhibits neointimal hyperplasia and smooth muscle proliferation. However, its role in addition to dual antiplatelet therapy (DAPT) of aspirin and clopidogrel in patients undergoing percutaneous coronary intervention (PCl) is not well defined.

What does this study add?

- In patients undergoing $\mathrm{PCl}$, addition of cilostazol to DAPT results in decreased platelet reactivity and a significant reduction in cardiovascular outcomes including stent thrombosis, even in the drug-eluting stent era.

How might this impact on clinical practice?

- The current study provides evidence to support use of cilostazol as an attractive and strong competitor for newer antiplatelet regimens and should be evaluated in future trials in patients undergoing $\mathrm{PCl}$.

\section{INTRODUCTION}

Dual antiplatelet therapy (DAPT) with aspirin and an ADP receptor inhibitor is the standard of care for patients undergoing percutaneous coronary intervention (PCI). However, there is significant interindividual variability in the extent of platelet inhibition achieved with clopidogrel. ${ }^{1-3}$ Several studies have shown a correlation between high levels of on-treatment platelet reactivity (HTPR) and adverse cardiovascular outcomes, such that patients with HTPR (also called clopidogrel resistance) have a threefold to fivefold increased risk for recurrent ischaemic events. ${ }^{4}{ }^{5}$ Cilostazol, a phosphodiesterase III inhibitor, exhibits its antiplatelet effects via inhibition of the conversion of cyclic AMP (cAMP) to 5'-AMP 
causing a subsequent increase in cAMP within platelets, and has been shown to augment platelet inhibition when it is added to aspirin and clopidogrel as part of a triple therapy regimen. ${ }^{6}$ In addition, cilostazol inhibits neointimal hyperplasia and smooth muscle proliferation, and has the potential to reduce the risk of restenosis after coronary stent implantation. ${ }^{8-11}$ Despite these pharmacologic effects, clinical results from observational and small randomised trials have not shown a consistent clinical benefit.

Our objective was to evaluate whether triple antiplatelet therapy (TAPT) with cilostazol (in addition to aspirin and clopidogrel) decreases platelet reactivity and reduces adverse cardiovascular (CV) outcomes when compared with a dual antiplatelet (DAPT) regimen of aspirin and clopidogrel alone.

\section{METHODS}

\section{Eligibility criteria}

We conducted a MEDLINE, EMBASE and CENTRAL search using the MeSH terms 'cilostazol' and 'randomised clinical trial'. We limited our search to trials involving human subjects through May 2014. The search terms were broad with no language restrictions imposed. We checked the reference lists of review articles and prior meta-analyses to assess for additional eligible studies. Corresponding authors of studies were contacted for further information if relevant data were not reported. Trials in abstract format without a manuscript published were also included in the analysis.

To be included for analysis, eligible trials had to fulfil the following criteria: (1) randomised clinical trials of TAPT (aspirin, clopidogrel and cilostazol) in comparison to DAPT (aspirin and clopidogrel); (2) enrolment of patients undergoing PCI with drug-eluting or bare metal stents and (3) follow-up of at least 2 weeks for trials reporting platelet reactivity outcomes and at least 1 month for trials reporting cardiovascular outcomes.

\section{Selection and quality assessment}

Three authors (AS, BT and SB) independently reviewed trial eligibility and quality. Disagreements were resolved by consensus. Risk of bias was assessed using criteria recommended by the Cochrane Collaboration, specifically evaluating sequence generation of allocation; allocation concealment; blinding of participants, staff and outcome assessors; incomplete outcome data; selective outcome reporting; and other sources of bias. ${ }^{12}$ Trials with high or unclear risk of bias for the first three criteria were considered as high bias risk trials and the rest as low bias risk trials.

\section{Data extraction and synthesis}

The primary platelet reactivity outcome was differences in platelet reactivity unit (PRU) after treatment in TAPT versus DAPT groups. Secondary outcomes were percent platelet inhibition and rate of HTPR. We used a cut-off of PRU >235 as the threshold for identifying patients with HTPR who may be at high risk for ischaemic or thrombotic events following PCI, as has been recommended by a recent consensus document. ${ }^{13}$ Of note, definition of HTPR differed by study.

Our primary CV outcome was major adverse cardiovascular events (MACE), defined as death, myocardial infarction (MI) or target lesion revascularisation (TLR). We evaluated secondary CV outcomes of death, cardiovascular death, MI, stent thrombosis, TLR and target vessel revascularisation (TVR). Safety outcomes of major bleeding, minor bleeding, any (major or minor) bleeding and drug discontinuation due to adverse effects were also evaluated. The definitions of bleeding varied between the trials. Given the lack of consistent reporting of the Academic Research Consortium definitions of stent thrombosis from the studies, we used the individual trial protocol definitions of stent thrombosis.

\section{Statistical analysis}

We performed an intention to treat meta-analysis in line with recommendations from the Cochrane Collaboration and the PRISMA Statement ${ }^{14}{ }^{15}$ and used standard software for statistical analysis (STATA V.9.0, STATA Corp, Texas, USA). Heterogeneity was assessed using the $\mathrm{I}^{2}$ statistic, defined as the proportion of total variation observed between the trials attributable to differences between trials rather than sampling error (chance), with values $<25 \%$ considered as low and $>75 \%$ as high. ${ }^{16}$ The pooled effect for each grouping of trials was derived from the point estimate for each separate trial weighted by the inverse of the variance $\left(1 / \mathrm{SE}^{2}\right)$. Continuous variable outcomes (PRU, per cent platelet inhibition) between the groups were compared with both a fixed effect model using the inverse variance method and a random effects model using the DerSimonian and Laird method. For cardiovascular outcomes, rates were expressed per patient-years to adjust for the varying duration of follow-up. Results were therefore reported as incident rate ratios (IRR) and 95\% CIs with the use of both a fixed effect model using the method of Mantel and Haenszel and a random effects model using the method of DerSimonian and Laird, with the estimate of heterogeneity being taken from the Mantel-Haenszel model. Publication bias was estimated using the weighted regression tests of Begg and Egger. ${ }^{12}$

For platelet reactivity indices, analyses were stratified based on whether standard-dose $(75 \mathrm{mg})$ or high-dose $(150 \mathrm{mg})$ clopidogrel was used in the DAPT arm. In addition, further sensitivity analyses were performed based on the cohort enrolled: (1) acute coronary syndrome (ACS) versus not; and (2) enrolment of patients with HTPR at baseline versus not. For cardiovascular outcomes, analyses were stratified based on stent typedrug eluting stent (DES) versus Bare metal stent (BMS). A $p$ value of $<0.05$ was considered significant. 
Figure 1 Study selection.

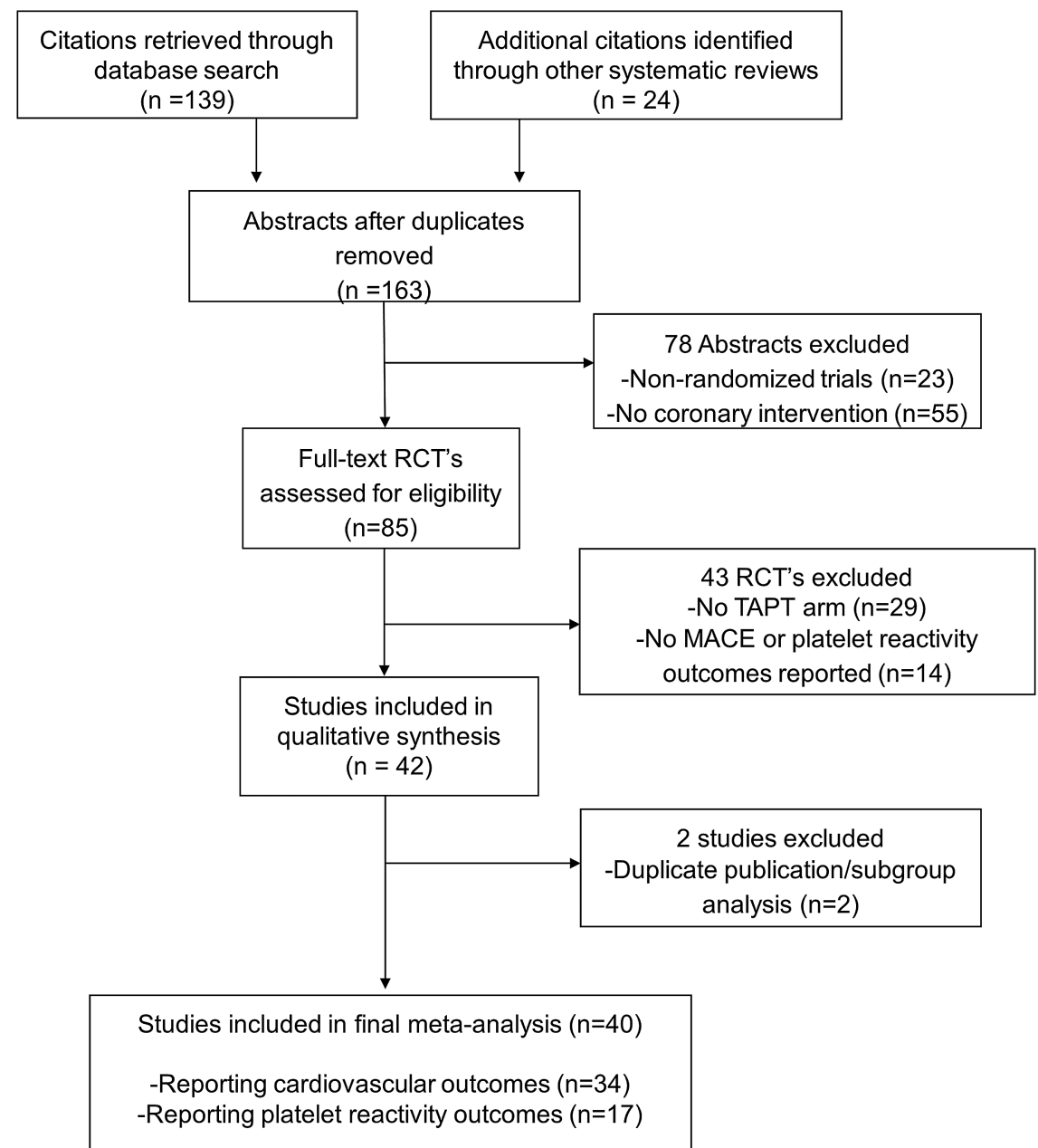

\section{RESULTS}

\section{Study selection}

We identified 41 trials that satisfied the inclusion criteria (figure 1). Seventeen trials reported platelet reactivity outcomes of which 10 comparator arms used high dose (150 mg) of clopidogrel. A total of 34 trials reported CV outcomes, the majority (25 trials) of which used DES.

\section{Baseline characteristics}

The baseline characteristics, inclusion criteria and quality assessment are summarised in tables 1-4. In order to quantify platelet reactivity outcomes, we evaluated 17 trials with 20 comparator arms and 5056 patients. The median follow-up was 30 days and although the definition of HTPR was heterogeneous, all trials used the VerifyNow P2Y12 assay to measure platelet reactivity. The analysis of cardiovascular outcomes included 34 trials with 14119 patients. The mean age of study participants was between 56.3 and 67.5 years, $37.9 \%$ of the patients had diabetes and the majority (77.6\%) underwent PCI with DES.

\section{Primary platelet reactivity outcomes}

Primary outcome: differences in PRU

TAPT resulted in a mean PRU reduction of $47.73(95 \%$ CI -61.41 to -34.04, $\mathrm{p}<0.0001$; mean PRU 182.90 vs 232.65) compared with DAPT (figure 2A). There was a larger mean difference between the TAPT and DAPT groups when the analysis was restricted to a DAPT group using standard-dose clopidogrel (mean PRU 189.54 vs 255.83) where the PRU value was lower by a mean of 64.10 (95\% CI -84.35 to -43.85$)$. Moreover, TAPT was associated with a lower PRU value even when compared with DAPT using high-dose clopidogrel (mean difference of 27.17) (mean PRU 176.27 vs 209.48) (figure 2A). The results were similar when stratified by ACS status (see web appendix figure A1) or by baseline clopidogrel resistance status (see web appendix figure A2). There was moderate-to-high heterogeneity for the above analysis. However, the heterogeneity was reduced in subgroup analysis restricted to comparison with high-dose clopidogrel (figure 2A), in trials enrolling patients with baseline clopidogrel resistance (see web appendix figure A2 and in trials enrolling patients without ACS (see web appendix figure A1).

In addition, the mean PRU values on treatment in the TAPT group in each of the trials were below a PRU of 235, which has been cited in the literature as the suggested threshold for defining HTPR. ${ }^{13}$

Secondary outcomes: percent platelet inhibition and high on-treatment platelet reactivity

TAPT was associated with a $12.71 \%$ greater platelet inhibition compared to DAPT for the overall cohort 
Table 1 Baseline characteristics of included trials for platelet reactivity outcomes

\begin{tabular}{|c|c|c|c|c|c|c|}
\hline Trial & Year & $\mathbf{N}$ & Comparison & $\begin{array}{l}\text { SD or HD } \\
\text { (DAPT group) }\end{array}$ & $\begin{array}{l}\text { Mean age } \\
\text { (years) }\end{array}$ & $\begin{array}{l}\text { Follow-up } \\
\text { (days) }\end{array}$ \\
\hline ACCEL-AMI ${ }^{29}$ & 2009 & 90 & $\begin{array}{l}\text { Aspirin/clopidogrel/cilostazol } \\
\text { vs aspirin/clopidogrel }\end{array}$ & Both & 62 & 30 \\
\hline ACCEL-LOADING-ACS ${ }^{30}$ & 2012 & 218 & $\begin{array}{l}\text { Aspirin/clopidogrel/cilostazol } \\
\text { vs aspirin/clopidogrel }\end{array}$ & SD & 63 & 30 \\
\hline ACCEL-POLYMORPHISM ${ }^{31}$ & 2010 & 134 & $\begin{array}{l}\text { Aspirin/clopidogrel/cilostazol } \\
\text { vs aspirin/clopidogrel }\end{array}$ & HD & 63 & 30 \\
\hline ACCEL-PPI ${ }^{32}$ & 2012 & 90 & $\begin{array}{l}\text { Aspirin/clopidogrel/cilostazol } \\
\text { vs aspirin/clopidogrel }\end{array}$ & HD & NR & 30 \\
\hline ACCEL-RESISTANCE ${ }^{33}$ & 2009 & 60 & $\begin{array}{l}\text { Aspirin/clopidogrel/cilostazol } \\
\text { vs aspirin/clopidogrel }\end{array}$ & HD & 63 & 30 \\
\hline CILON-T ${ }^{34}$ & 2011 & 716 & $\begin{array}{l}\text { Aspirin/clopidogrel/cilostazol } \\
\text { vs aspirin/clopidogrel }\end{array}$ & SD & 64 & 180 \\
\hline Gao et $a \beta^{\beta 5}$ & 2013 & 428 & $\begin{array}{l}\text { Aspirin/clopidogrel/cilostazol } \\
\text { vs aspirin/clopidogrel }\end{array}$ & SD & 56 & 365 \\
\hline Guan et $a l^{\beta 6}$ & 2012 & 840 & $\begin{array}{l}\text { Aspirin/clopidogrel/cilostazol } \\
\text { vs aspirin/clopidogrel }\end{array}$ & SD & 60 & 30 \\
\hline HOST-ASSURE ${ }^{37}$ & 2013 & 1356 & $\begin{array}{l}\text { Aspirin/clopidogrel/cilostazol } \\
\text { vs aspirin/clopidogrel }\end{array}$ & Both & 63 & 30 \\
\hline Jeong et $\left.a\right|^{38}$ & 2014 & 275 & $\begin{array}{l}\text { Aspirin/clopidogrel/cilostazol } \\
\text { vs aspirin/clopidogrel }\end{array}$ & Both & NR & 30 \\
\hline Jin et $a \beta^{\beta 9}$ & 2012 & 60 & $\begin{array}{l}\text { Aspirin/clopidogrel/cilostazol } \\
\text { vs aspirin/clopidogrel }\end{array}$ & $\mathrm{HD}$ & 62 & 30 \\
\hline Kim et $a f^{40}$ & 2011 & 126 & $\begin{array}{l}\text { Aspirin/clopidogrel/cilostazol } \\
\text { vs aspirin/clopidogrel }\end{array}$ & HD & 62 & 30 \\
\hline Kim et $\left.a\right|^{41}$ & 2007 & 60 & $\begin{array}{l}\text { Aspirin/clopidogrel/cilostazol } \\
\text { vs aspirin/clopidogrel }\end{array}$ & SD & 63 & 30 \\
\hline Kum et $a l^{42}$ & 2009 & 66 & $\begin{array}{l}\text { Aspirin/clopidogrel/cilostazol } \\
\text { vs aspirin/clopidogrel }\end{array}$ & SD & 62 & 14 \\
\hline Lee et $a I^{43}$ & 2010 & 63 & $\begin{array}{l}\text { Aspirin/clopidogrel/cilostazol } \\
\text { vs aspirin/clopidogrel }\end{array}$ & Both & NR & 14 \\
\hline PIANO-2 CKD ${ }^{44}$ & 2011 & 74 & $\begin{array}{l}\text { Aspirin/clopidogrel/cilostazol } \\
\text { vs aspirin/clopidogrel }\end{array}$ & Both & 53 & 14 \\
\hline Shim et $a{ }^{45}$ & 2009 & 379 & $\begin{array}{l}\text { Aspirin/clopidogrel/cilostazol } \\
\text { vs aspirin/clopidogrel }\end{array}$ & SD & 61 & 14 \\
\hline \multicolumn{7}{|c|}{$\begin{array}{l}\text { ACCEL-AMI, adjunctive Cilostazol versus High Maintenance Dose Clopidogrel in patients with AMI; ACCEL-LOADING-ACS, Multicentre } \\
\text { Randomised Trial Evaluating Efficacy of Cilostazol on Platelet Aggregation, Inflammation and Myonecrosis in ACS Patients; } \\
\text { ACCEL-POLYMORPHISM, Cytochrome 2C19 Polymorphism and Response to Adjunctive Cilostazol versus High Maintenance-Dose } \\
\text { Clopidogrel in Patients Undergoing Percutaneous Coronary Intervention; ACCEL-PPI, Pharmacodynamics Effects of Adding Cilostazol versus } \\
\text { Double-dose Clopidogrel in Patients with Acute Myocardial Infarction During Proton Pump Inhibitor Co-administration; ACCEL-RESISTANCE, } \\
\text { Adjunctive Cilostazol Versus High Maintenance Dose Clopidogrel in Patients with Clopidogrel Resistance; CILON-T, Influence of } \\
\text { Cilostazol-based Triple Antiplatelet Therapy on Ischaemic Complication After Drug-eluting Stent Implantation; HD, high-dose clopidogrel } \\
\text { (150 mg); HOST-ASSURE, Harmonising Optimal Strategy for Treatment of Coronary Artery Stenosis-Safety and Effectiveness of } \\
\text { Drug-Eluting Stents and Antiplatelet Regimen; NR, not reported; PIANO-2 CKD, Platelet Reactivity in Patients with Chronic Kidney Disease } \\
\text { Receiving Adjunctive Cilostazol Compared with a High-Maintenance Dose of Clopidogrel; SD, Standard-dose clopidogrel (75 mg). }\end{array}$} \\
\hline
\end{tabular}

(95\% CI 10.76 to $14.67, \mathrm{p}<0.0001$ ) (figure $2 \mathrm{~B}$ ). TAPT was also associated with a greater platelet inhibition in comparison with DAPT using standard-dose clopidogrel (14.37\% mean greater platelet inhibition) and remained significant even when compared with DAPT using highdose clopidogrel $(9.07 \%$ mean greater platelet inhibition) (figure 2B). There was moderate heterogeneity for the above analysis. The results were similar when stratified by ACS status (see web appendix figure A3) or by baseline clopidogrel resistance status (see web appendix figure A4).
In addition, TAPT was associated with a $60 \%$ reduction in the risk of HTPR when compared with DAPT (figure 2C) (relative risk $=0.40 ; 95 \%$ CI 0.30 to 0.53 , $\mathrm{p}<0.0001$ ). When stratified by clopidogrel dose, TAPT was associated with a $50 \%$ reduction in risk of HTPR compared to standard-dose DAPT and a $72 \%$ reduction compared to high-dose DAPT (figure 2C). Heterogeneity was moderate with no evidence for significant publication bias. The results were similar when stratified by ACS status (see web appendix figure A5) or by baseline clopidogrel resistance status (see web appendix figure A6). 
Table 2 Inclusion criteria and study quality for platelet reactivity outcomes trials

\begin{tabular}{|c|c|c|c|c|}
\hline Trial & Cohort & Definition of HTPR & $\begin{array}{l}\text { Platelet } \\
\text { reactivity } \\
\text { assay }\end{array}$ & $\begin{array}{l}\text { Quality of } \\
\text { study }^{\star}\end{array}$ \\
\hline ACCEL-AMI $^{29}$ & $\begin{array}{l}\text { Patients with ACS undergoing } \\
\mathrm{PCI}\end{array}$ & $\begin{array}{l}5 \text { and } 20 \mu \mathrm{M} \text { ADP-induced } \\
\text { maximal platelet aggregation } \\
>50 \%\end{array}$ & $\begin{array}{l}\text { VerifyNow } \\
\text { P2Y12; LTA }\end{array}$ & +++ \\
\hline ACCEL-LOADING-ACS ${ }^{30}$ & $\begin{array}{l}\text { Patients with non-ST-elevation MI } \\
\text { undergoing } \mathrm{PCl}\end{array}$ & NR & $\begin{array}{l}\text { VerifyNow } \\
\text { P2Y12 }\end{array}$ & \pm \pm \pm \\
\hline ACCEL-POLYMORPHISM ${ }^{31}$ & $\begin{array}{l}\text { Patients with high post-treatment } \\
\text { platelet reactivity or diabetes } \\
\text { undergoing } \mathrm{PCl}\end{array}$ & $\begin{array}{l}5 \mu \mathrm{M} \text { ADP-induced maximal } \\
\text { platelet aggregation }>50 \%\end{array}$ & $\begin{array}{l}\text { VerifyNow } \\
\text { P2Y12; LTA }\end{array}$ & +++ \\
\hline ACCEL-PPI ${ }^{32}$ & $\begin{array}{l}\text { Patients with acute } \mathrm{MI} \\
\text { undergoing } \mathrm{PCl}\end{array}$ & $\begin{array}{l}20 \mu \mathrm{M} \text { ADP-induced } \\
\text { maximal platelet aggregation } \\
>59 \%\end{array}$ & LTA & \pm \pm \pm \\
\hline ACCEL-RESISTANCE ${ }^{33}$ & $\begin{array}{l}\text { Patients with high on-treatment } \\
\text { platelet reactivity undergoing } \mathrm{PCI}\end{array}$ & $\begin{array}{l}5 \mu \mathrm{M} \text { ADP-induced maximal } \\
\text { platelet aggregation }>50 \%\end{array}$ & $\begin{array}{l}\text { VerifyNow } \\
\text { P2Y12; LTA }\end{array}$ & ++ \pm \\
\hline CILON-T ${ }^{34}$ & $\begin{array}{l}\text { Patients with angina undergoing } \\
\mathrm{PCl}\end{array}$ & NR & $\begin{array}{l}\text { VerifyNow } \\
\text { P2Y12 }\end{array}$ & ++ \pm \\
\hline Gao et $a{ }^{\beta 5}$ & Obese patients undergoing $\mathrm{PCl}$ & $\begin{array}{l}\text { Post-treatment platelet } \\
\text { aggregation absolute } \\
\text { difference } 10 \% \text { or less }\end{array}$ & LTA & \pm \pm \pm \\
\hline Guan et $a l^{\beta 6}$ & $\begin{array}{l}\text { Patients with } \mathrm{ACS} \text { and high } \\
\text { on-treatment platelet reactivity } \\
\text { undergoing } \mathrm{PCl}\end{array}$ & $\begin{array}{l}20 \mu \mathrm{M} \text { ADP-induced } \\
\text { maximal platelet aggregation } \\
>55 \%\end{array}$ & LTA & \pm \pm \pm \\
\hline HOST-ASSURE ${ }^{37}$ & $\begin{array}{l}\text { All-comer patients undergoing } \\
\mathrm{PCI}\end{array}$ & NR & $\begin{array}{l}\text { VerifyNow } \\
\text { P2Y12 }\end{array}$ & \pm \pm+ \\
\hline Jeong et $a{ }^{\beta 8}$ & $\begin{array}{l}\text { Patients with ACS undergoing } \\
\mathrm{PCI}\end{array}$ & NR & LTA & \pm \pm \pm \\
\hline Jin et $a l^{\beta 9}$ & Patients undergoing $\mathrm{PCl}$ & $\%$ platelet inhibition $<20$ & $\begin{array}{l}\text { VerifyNow } \\
\text { P2Y12; LTA }\end{array}$ & \pm++ \\
\hline Kim et $a f^{40}$ & $\begin{array}{l}\text { Patients with acute } \mathrm{MI} \\
\text { undergoing } \mathrm{PCl}\end{array}$ & $\begin{array}{l}20 \mu \mathrm{M} \text { ADP-induced } \\
\text { maximal platelet aggregation } \\
>59 \%\end{array}$ & $\begin{array}{l}\text { VerifyNow } \\
\text { P2Y12; LTA }\end{array}$ & ++ \pm \\
\hline Kim et $a{ }^{41}$ & $\begin{array}{l}\text { Patients with } \mathrm{ST} \text {-elevation } \mathrm{Ml} \\
\text { undergoing } \mathrm{PCl}\end{array}$ & $\%$ platelet inhibition $<20$ & $\begin{array}{l}\text { VerifyNow } \\
\text { P2Y12; LTA }\end{array}$ & \pm \pm \pm \\
\hline Kum et $a l^{42}$ & Patients undergoing $\mathrm{PCl}$ & NR & $\begin{array}{l}\text { VerifyNow } \\
\text { P2Y12 }\end{array}$ & \pm \pm \pm \\
\hline Lee et $a{ }^{43}$ & $\begin{array}{l}\text { Patients with high on-treatment } \\
\text { platelet reactivity undergoing } \mathrm{PCI}\end{array}$ & $\%$ platelet inhibition $<20$ & $\begin{array}{l}\text { VerifyNow } \\
\text { P2Y12 }\end{array}$ & + \pm \pm \\
\hline PIANO-2 CKD ${ }^{44}$ & $\begin{array}{l}\text { Patients with renal disease on } \\
\text { haemodialysis undergoing } \mathrm{PCl}\end{array}$ & $\begin{array}{l}5 \mu \mathrm{M} \text { ADP-induced maximal } \\
\text { platelet aggregation }>50 \%\end{array}$ & $\begin{array}{l}\text { VerifyNow } \\
\text { P2Y12; LTA }\end{array}$ & +++ \\
\hline Shim et $a f^{45}$ & $\begin{array}{l}\text { Patients undergoing } \mathrm{PCl} \text { with } \\
\text { DES }\end{array}$ & $\%$ platelet inhibition $<20$ & $\begin{array}{l}\text { VerifyNow } \\
\text { P2Y12 }\end{array}$ & + \pm \pm \\
\hline
\end{tabular}

*Represents risk of bias based on: sequence generation of allocation; allocation concealment and blinding. ' + ' represents low bias risk, '-' high bias risk and ' \pm ' unclear bias risk.

ACCEL-AMI, adjunctive Cilostazol versus High Maintenance Dose Clopidogrel in patients with AMI; ACCEL-LOADING-ACS, Multicentre Randomised Trial Evaluating Efficacy of Cilostazol on Platelet Aggregation, Inflammation and Myonecrosis in ACS Patients;

ACCEL-POLYMORPHISM, Cytochrome 2C19 Polymorphism and Response to Adjunctive Cilostazol versus High Maintenance-Dose Clopidogrel in Patients Undergoing Percutaneous Coronary Intervention; ACCEL-PPI, Pharmacodynamics Effects of Adding Cilostazol versus Double-dose Clopidogrel in Patients with Acute Myocardial Infarction During Proton Pump Inhibitor Co-administration; ACCEL-RESISTANCE, Adjunctive Cilostazol Versus High Maintenance Dose Clopidogrel in Patients with Clopidogrel Resistance; ACS, acute coronary syndrome; CILON-T, Influence of Cilostazol-based Triple Antiplatelet Therapy on Ischaemic Complication After Drug-eluting Stent Implantation; HD, high-dose clopidogrel $(150 \mathrm{mg})$; HOST-ASSURE, Harmonising Optimal Strategy for Treatment of Coronary Artery Stenosis-Safety and Effectiveness of Drug-Eluting Stents and Antiplatelet Regimen; LTA, light transmittance aggregometry; MI, myocardial infarction; NR, not reported; PIANO-2 CKD, Platelet Reactivity in Patients with Chronic Kidney Disease Receiving Adjunctive Cilostazol Compared with a High-Maintenance Dose of Clopidogrel; SD, Standard-dose clopidogrel (75 mg).

\section{Cardiovascular outcomes}

Primary outcome

TAPT was associated with a $32 \%$ reduction in the risk of MACE (IRR $=0.68 ; 95 \%$ CI 0.60 to 0.78$)$ when compared with DAPT for the overall cohort (figure 3A). This effect was observed regardless of stent type $\left(\mathrm{P}_{\text {interaction }}\right.$ $>0.05)$ such that even in patients undergoing PCI with DES, TAPT resulted in a $36 \%$ reduction in MACE 
Table 3 Baseline characteristics of included trials for cardiovascular outcomes

\begin{tabular}{|c|c|c|c|c|c|c|c|c|}
\hline Trial & Year & $\mathrm{N}$ & Comparison & $\begin{array}{l}\text { Follow-up } \\
\text { (months) }\end{array}$ & $\begin{array}{l}\text { Mean } \\
\text { age } \\
\text { (years) }\end{array}$ & $\begin{array}{l}\text { DM } \\
(\%)\end{array}$ & Stent type & $\begin{array}{l}\text { DES } \\
(\%)\end{array}$ \\
\hline $\mathrm{ABCD}^{46}$ & 2014 & 630 & $\begin{array}{l}\text { Aspirin/clopidogrel/cilostazol } \\
\text { vs aspirin/clopidogrel }\end{array}$ & 12 & 65 & 31 & BES & 100 \\
\hline ACCEL-AMI ${ }^{29}$ & 2010 & 90 & $\begin{array}{l}\text { Aspirin/clopidogrel/cilostazol } \\
\text { vs aspirin/clopidogrel }\end{array}$ & 1 & 62 & 21 & PES $>S E S>Z E S$ & 100 \\
\hline $\begin{array}{l}\text { ACCEL- } \\
\text { LOADING- } \\
\text { ACS }^{30}\end{array}$ & 2012 & 218 & $\begin{array}{l}\text { Aspirin/clopidogrel/cilostazol } \\
\text { vs aspirin/clopidogrel }\end{array}$ & 1 & 63 & 23 & DES, BMS & 95 \\
\hline $\begin{array}{l}\text { ACCEL- } \\
\text { RESISTANCE }\end{array}$ & 2009 & 60 & $\begin{array}{l}\text { Aspirin/clopidogrel/cilostazol } \\
\text { vs aspirin/high-dose } \\
\text { clopidogrel }\end{array}$ & 1 & 63 & 23 & DES & 100 \\
\hline Ahn $\mathrm{CM}$ et $a{ }^{47}$ & 2011 & 130 & $\begin{array}{l}\text { Aspirin/clopidogrel/cilostazol } \\
\text { vs aspirin/clopidogrel }\end{array}$ & 24 & 64 & 22 & SES & 100 \\
\hline Chen YD et $a l^{48}$ & 2006 & 120 & $\begin{array}{l}\text { Aspirin/clopidogrel/cilostazol } \\
\text { vs aspirin/clopidogrel }\end{array}$ & 9 & 58 & 30 & BMS & 0 \\
\hline CIDES $^{49}$ & 2008 & 280 & $\begin{array}{l}\text { Aspirin/clopidogrel/cilostazol } \\
\text { vs aspirin/clopidogrel }\end{array}$ & 6 & 62 & 100 & PES, SES & 100 \\
\hline CILON-T ${ }^{34}$ & 2011 & 960 & $\begin{array}{l}\text { Aspirin/clopidogrel/cilostazol } \\
\text { vs aspirin/clopidogrel }\end{array}$ & 6 & 64 & 34 & PES, ZES & 100 \\
\hline CLEAR $^{50}$ & 2011 & 120 & $\begin{array}{l}\text { Aspirin/clopidogrel/cilostazol } \\
\text { vs aspirin/clopidogrel }\end{array}$ & 6 & 66 & 42 & SES $>Z E S>P E S>E E S$ & 100 \\
\hline CREST $^{51}$ & 2005 & 705 & $\begin{array}{l}\text { Aspirin/clopidogrel/cilostazol } \\
\text { vs aspirin/clopidogrel }\end{array}$ & 6 & 60 & 26 & BMS & 0 \\
\hline $\begin{array}{l}\text { DECLARE- } \\
\text { DIABETES }^{52} 53\end{array}$ & $\begin{array}{l}2008 / \\
2010\end{array}$ & 450 & $\begin{array}{l}\text { Aspirin/clopidogrel/cilostazol } \\
\text { vs aspirin/clopidogrel }\end{array}$ & 24 & 61 & 100 & PES, SES & 100 \\
\hline $\begin{array}{l}\text { DECLARE- } \\
\text { LONG }^{53} 54\end{array}$ & $\begin{array}{l}2007 / \\
2010\end{array}$ & 450 & $\begin{array}{l}\text { Aspirin/clopidogrel/cilostazol } \\
\text { vs aspirin/clopidogrel }\end{array}$ & 24 & 61 & 33 & PES, SES & 100 \\
\hline $\begin{array}{l}\text { DECLARE- } \\
\text { LONG } \|^{55}\end{array}$ & 2011 & 499 & $\begin{array}{l}\text { Aspirin/clopidogrel/cilostazol } \\
\text { vs aspirin/clopidogrel }\end{array}$ & 12 & 62 & 35 & ZES & 100 \\
\hline Gao et $a \beta^{35}$ & 2013 & 428 & $\begin{array}{l}\text { Aspirin/clopidogrel/cilostazol } \\
\text { vs aspirin/clopidogrel }\end{array}$ & 12 & 56 & 18 & SES $>$ PES & 100 \\
\hline Guan et $a^{\beta 6}$ & 2012 & 840 & $\begin{array}{l}\text { Aspirin/clopidogrel/cilostazol } \\
\text { vs aspirin/clopidogrel }\end{array}$ & 1 & 60 & NR & DES & 100 \\
\hline Han et $a^{p^{6}}$ & 2009 & 1212 & $\begin{array}{l}\text { Aspirin/clopidogrel/cilostazol } \\
\text { vs aspirin/clopidogrel }\end{array}$ & 12 & 60 & 22 & BMS, DES & 52 \\
\hline Han et $a p^{7}$ & 2006 & 120 & $\begin{array}{l}\text { Aspirin/clopidogrel/cilostazol } \\
\text { vs aspirin/clopidogrel }\end{array}$ & 3 & 61 & 23 & BMS, DES & 43 \\
\hline $\begin{array}{l}\text { HOST- } \\
\text { ASSURE }^{37}\end{array}$ & 2013 & 3755 & $\begin{array}{l}\text { Aspirin/clopidogrel/cilostazol } \\
\text { vs aspirin/clopidogrel }\end{array}$ & 1 & 63 & 32 & ZES-R>EES-PtCr & 100 \\
\hline Hu et $a^{58}$ & 2013 & 146 & $\begin{array}{l}\text { Aspirin/clopidogrel/cilostazol } \\
\text { vs aspirin/clopidogrel }\end{array}$ & 12 & 63 & NR & NR & NR \\
\hline Jin et $a \beta^{\beta 9}$ & 2012 & 60 & $\begin{array}{l}\text { Aspirin/clopidogrel/cilostazol } \\
\text { vs aspirin/clopidogrel }\end{array}$ & 1 & 62 & 45 & DES & 100 \\
\hline Kim et $a^{59}$ & 2008 & 109 & $\begin{array}{l}\text { Aspirin/clopidogrel/cilostazol } \\
\text { vs aspirin/clopidogrel }\end{array}$ & 6 & 68 & 29 & PES>SES & 100 \\
\hline Kim et $a l^{41}$ & 2007 & 60 & $\begin{array}{l}\text { Aspirin/clopidogrel/cilostazol } \\
\text { vs aspirin/clopidogrel }\end{array}$ & 1 & 63 & 29 & SES>PES>others & 100 \\
\hline Kum et $a l^{42}$ & 2009 & 603 & $\begin{array}{l}\text { Aspirin/clopidogrel/cilostazol } \\
\text { vs aspirin/clopidogrel }\end{array}$ & 6 & 62 & 26 & DES & 100 \\
\hline Lee et $a^{60}$ & 2007 & 20 & $\begin{array}{l}\text { Aspirin/clopidogrel/cilostazol } \\
\text { vs aspirin/clopidogrel }\end{array}$ & 1 & 56 & 25 & NR & 100 \\
\hline $\begin{array}{l}\text { LONG- } \\
\text { DES-II61 } 62\end{array}$ & 2007 & 500 & $\begin{array}{l}\text { Aspirin/clopidogrel/cilostazol } \\
\text { vs aspirin/clopidogrel }\end{array}$ & 9 & 61 & 33 & PES, SES & 100 \\
\hline Lu et $a^{{ }^{3}}$ & 2006 & 120 & $\begin{array}{l}\text { Aspirin/clopidogrel/cilostazol } \\
\text { vs aspirin/clopidogrel }\end{array}$ & $6-9$ & 71 & NR & BMS & 0 \\
\hline Lu et $a^{64}$ & 2007 & 402 & $\begin{array}{l}\text { Aspirin/clopidogrel/cilostazol } \\
\text { vs aspirin/clopidogrel }\end{array}$ & 6 & 61 & 44 & BMS, DES & 85 \\
\hline
\end{tabular}


Table 3 Continued

\begin{tabular}{|c|c|c|c|c|c|c|c|c|}
\hline Trial & Year & $\mathbf{N}$ & Comparison & $\begin{array}{l}\text { Follow-up } \\
\text { (months) }\end{array}$ & $\begin{array}{l}\text { Mean } \\
\text { age } \\
\text { (years) }\end{array}$ & $\begin{array}{l}\text { DM } \\
(\%)\end{array}$ & Stent type & $\begin{array}{l}\text { DES } \\
(\%)\end{array}$ \\
\hline Min et $a l^{10}$ & 2007 & 59 & $\begin{array}{l}\text { Aspirin/clopidogrel or } \\
\text { ticlopidine/cilostazol vs } \\
\text { aspirin/clopidogrel or } \\
\text { ticlopidine }\end{array}$ & 6 & 62 & 26 & BMS & 0 \\
\hline OPTIMUS-2 ${ }^{6}$ & 2008 & 50 & $\begin{array}{l}\text { Aspirin/clopidogrel/cilostazol } \\
\text { vs aspirin/clopidogrel }\end{array}$ & 1 & 64 & 100 & NR & 100 \\
\hline Shen et $a{ }^{65}$ & 2010 & 160 & $\begin{array}{l}\text { Aspirin/Clopidogrel/ } \\
\text { Cilostazol vs Aspirin/ } \\
\text { Clopidogrel }\end{array}$ & 12 & 69 & 100 & DES & 100 \\
\hline Suh et $a^{{ }^{6}}$ & 2009 & 143 & $\begin{array}{l}\text { Aspirin/clopidogrel/cilostazol } \\
\text { vs aspirin/clopidogrel }\end{array}$ & 25 & 62 & 100 & PES>SES & 100 \\
\hline Wang et $a^{67}$ & 2005 & 193 & $\begin{array}{l}\text { Aspirin/clopidogrel/cilostazol } \\
\text { vs aspirin/clopidogrel }\end{array}$ & 12 & 62 & 28 & BMS & 0 \\
\hline Wang et $a^{68}$ & 2010 & 164 & $\begin{array}{l}\text { Aspirin/clopidogrel/cilostazol } \\
\text { vs aspirin/clopidogrel }\end{array}$ & 12 & 68 & NR & BMS, DES & NR \\
\hline Zang et $a^{69}$ & 2008 & 263 & $\begin{array}{l}\text { Aspirin/clopidogrel/cilostazol } \\
\text { vs aspirin/clopidogrel }\end{array}$ & 12 & 59 & 100 & BMS, DES & 53 \\
\hline \multicolumn{9}{|c|}{$\begin{array}{l}\text { ABCD, Evaluating Additional Benefit of Cilostazol to Dual Antiplatelet Therapy in Patients with Long or Multivessel Coronary Artery Disease } \\
\text { underwent Biolimus-Eluting Stent Implantation; ACS, acute coronary syndrome; AMI, acute myocardial infarction; BES, biolimus-eluting stent; } \\
\text { BMS, bare metal stent; CIDES, comparison of cilostazol versus clopidogrel after drug-eluting stenting in diabetic patients; CILON-T, Influence } \\
\text { of Cilostazol-based Triple Antiplatelet Therapy on Ischaemic Complication After Drug-eluting Stent Implantation; CLEAR, The Cilostazol } \\
\text { Administration Before Percutaneous Coronary Intervention for Reduction of Periprocedural Myonecrosis Trial; CREST, Coronary Stent } \\
\text { Restenosis in Patients Treated with Cilostazol; DECLARE-LONG II: Triple Antiplatelet Therapy With Dual Antiplatelet Therapy to Reduce } \\
\text { Restenosis After Drug-Eluting Stent Implantation in Long Coronary Lesions; DECLARE-DIABETES, A Randomised Comparison of Triple } \\
\text { Antiplatelet Therapy with Dual Antiplatelet Therapy After Drug-Eluting Stent Implantation in Diabetic Patients; DECLARE-LONG, Drug-Eluting } \\
\text { Stenting Followed by Cilostazol Treatment Reduces Late Restenosis in Patients with Long Coronary Lesions; DES, drug-eluting stent; DM, } \\
\text { diabetes mellitus; EES, everolimus-eluting stent; EES-PtCr, everolimus-eluting platinum-chromium alloy stent; LONG-DES, Sirolimus-Eluting } \\
\text { Stent Versus Paclitaxel-Eluting Stent for Patients With Long Coronary Artery Disease; OPTIMUS-2, Impact of Cilostazol on Platelet Function } \\
\text { Profiles in Patients with Diabetes Mellitus and Coronary Artery Disease on Dual Antiplatelet Therapy; PES, Paclitaxel-eluting stent; SES, } \\
\text { Sirolimus-eluting stent; ZES, Zotarolimus-eluting stent; ZES-R, Zotarolimus-eluting Resolute stent. } \\
\text { Other trial expansions as in tables } 1 \text { and } 2 \text {. }\end{array}$} \\
\hline
\end{tabular}

(IRR $=0.64 ; 95 \%$ CI 0.55 to 0.75$)$ when compared with DAPT alone (figure 3A). There was low heterogeneity in the analysis and no evidence for significant publication bias.

\section{Secondary outcomes}

TAPT was associated with similar IRR for death (IRR=0.79; 95\% CI 0.58 to 1.09 ) (figure 3B), cardiovascular death $(\mathrm{IRR}=0.74 ; 95 \%$ CI 0.42 to 1.30$)$ and $\mathrm{MI}$ (IRR=0.85; 95\% CI 0.63 to 1.14 ) (figure 3C) for the overall cohort. The IRR was independent of stent type as TAPT showed benefit regardless whether BMS and DES was used (stent type, $P_{\text {interaction }}>0.05$ ). In the overall cohort, TAPT was associated with a $43 \%$ reduction in the risk of TLR (IRR=0.57; 95\% CI 0.44 to 0.73 ) (figure $3 \mathrm{D}$ ) and a $31 \%$ reduction in the risk of TVR (IRR $=0.69 ; 95 \%$ CI 0.59 to 0.81 ) (figure 3E) compared with DAPT. TAPT efficacy for reducing TLR and TVR was present even when the analyses were restricted to studies using DES. In DES-treated patients, TAPT resulted in a $43 \%$ reduction in TLR (IRR $=0.57 ; 95 \%$ CI 0.44 to 0.74 ) and a $35 \%$ reduction in TVR (IRR=0.65; $95 \%$ CI 0.54 to 0.79 ) with TAPT compared with DAPT.
TAPT was associated with significantly lower stent thrombosis rate when compared with DAPT $(\mathrm{IRR}=0.63$; $95 \%$ CI 0.40 to 0.98 ) (figure $3 \mathrm{~F}$ ). There was no heterogeneity $(0 \%)$ in all of the above analyses and no evidence for significant publication bias.

\section{Safety outcomes}

TAPT was associated with a numerically increased risk of major (IRR $=1.24 ; 95 \%$ CI 0.79 to 1.92 ) (figure $4 \mathrm{~A}$ ), minor $(\mathrm{IRR}=1.37 ; 95 \%$ CI 0.88 to 2.14 ) (figure $4 \mathrm{~B}$ ), or any bleeding (IRR=1.26; 95\% CI 0.99 to 1.61 ) (figure 4C) compared with DAPT, although these were not statistically significant. TAPT was also associated with a $59 \%$ increase in drug discontinuation due to adverse events (IRR=1.59; 95\% CI 1.32 to 1.91 ) (figure $4 \mathrm{D}$ ) when compared with DAPT. The most commonly listed causes for drug discontinuation were headache, skin rash and palpitations/tachycardia. There was no-to-modest (for drug discontinuation outcomes) heterogeneity in all of the above analyses and no evidence for significant publication bias. 
Table 4 Inclusion criteria and study quality of included cardiovascular outcomes trials

\begin{tabular}{|c|c|c|}
\hline Trial & Cohort & Quality of study* \\
\hline$A B C D^{46}$ & Patients with long or multivessel disease undergoing $\mathrm{PCl}$ & ++ \pm \\
\hline ACCEL-AMI ${ }^{29}$ & Patients with ACS undergoing $\mathrm{PCl}$ & +++ \\
\hline ACCEL-LOADING-ACS ${ }^{30}$ & Patients with non-ST-elevation $\mathrm{MI}$ undergoing $\mathrm{PCl}$ & \pm \pm \pm \\
\hline ACCEL-RESISTANCE ${ }^{33}$ & Patients with high on-treatment platelet reactivity undergoing $\mathrm{PCl}$ & ++ \pm \\
\hline Ahn et $a{ }^{47}$ & Patient with ACS undergoing $\mathrm{PCl}$ & \pm \pm+ \\
\hline Chen et $a^{48}$ & Patients with ACS undergoing $\mathrm{PCl}$ & \pm++ \\
\hline CIDES $^{49}$ & Patients with diabetes undergoing $\mathrm{PCl}$ & \pm \pm \pm \\
\hline CILON-T ${ }^{34}$ & Patients with angina undergoing $\mathrm{PCl}$ & ++ \pm \\
\hline CLEAR $^{50}$ & Patients with stable angina undergoing $\mathrm{PCl}$ & \pm \pm \pm \\
\hline CREST $^{51}$ & Patients with $\mathrm{ACS} /$ known stenosis undergoing $\mathrm{PCI}$ & +++ \\
\hline DECLARE-DIABETES ${ }^{52}$ & Patients with ACS and diabetes undergoing $\mathrm{PCI}$ & + \pm \pm \\
\hline DECLARE-LONG ${ }^{54}$ & Patients with ACS and stenosis of long $(>25 \mathrm{~mm}$ ) lesions undergoing $\mathrm{PCl}$ & + \pm \pm \\
\hline DECLARE-LONG $\|^{55}$ & Patients with ACS/known stenosis of long $(>25 \mathrm{~mm}$ ) lesions undergoing $\mathrm{PCl}$ & +++ \\
\hline Gao et $a{ }^{\beta 5}$ & Obese patients undergoing $\mathrm{PCl}$ & \pm \pm \pm \\
\hline Guan et $\left.a\right|^{\beta 6}$ & Patients with ACS and high on-treatment platelet reactivity undergoing $\mathrm{PCI}$ & \pm \pm \pm \\
\hline Han et $a^{56}$ & Patients with ACS undergoing $\mathrm{PCl}$ & ++ \pm \\
\hline Han et $a^{57}$ & Patients with ACS undergoing $\mathrm{PCI}$ & \pm \pm \pm \\
\hline HOST-ASSURE $^{37}$ & All-comer patients undergoing $\mathrm{PCl}$ & \pm \pm+ \\
\hline Hu et $a^{58}$ & Patients with ACS undergoing $\mathrm{PCl}$ & \pm \pm \pm \\
\hline Jin et $a{ }^{\beta 9}$ & Patients undergoing $\mathrm{PCl}$ & \pm++ \\
\hline Kim et $a^{59}$ & Patients with ACS/known stenosis undergoing $\mathrm{PCI}$ & \pm \pm \pm \\
\hline Kim et $a l^{41}$ & Patients with ST-elevation $\mathrm{MI}$ undergoing $\mathrm{PCl}$ & \pm \pm \pm \\
\hline Kum et $a l^{42}$ & Patients with ACS/known stenosis undergoing $\mathrm{PCI}$ & \pm \pm \pm \\
\hline Lee et $a f^{60}$ & Patients undergoing elective $\mathrm{PCl}$ & + \pm \pm \\
\hline LONG-DES-II ${ }^{61}$ & Patients with stenosis of long lesions undergoing $\mathrm{PCl}$ & ++ \pm \\
\hline Lu et $a l^{70}$ & Patients undergoing $\mathrm{PCl}$ & \pm \pm+ \\
\hline Lu et $a^{64}$ & Patients with ADP-induced platelet inhibition rates $<30 \%$ undergoing $\mathrm{PCl}$ & + \pm \pm \\
\hline Min et $a l^{10}$ & Patients with ACS/known stenosis undergoing elective $\mathrm{PCI}$ & \pm+ \pm \\
\hline OPTIMUS-2 6 & Patients with diabetes undergone $\mathrm{PCl}$ & +++ \\
\hline Shen et $a^{65}$ & Patients with ACS undergoing $\mathrm{PCl}$ & \pm \pm \pm \\
\hline Suh et $a^{66}$ & Patients with diabetes and chronic total occlusion undergoing $\mathrm{PCl}$ & \pm \pm \pm \\
\hline Wang et $a \rho^{7}$ & Patients with small vessel stenosis undergoing $\mathrm{PCl}$ & \pm \pm \pm \\
\hline Wang et $a^{68}$ & Patients with non-ST-elevation $\mathrm{Ml}$ undergoing $\mathrm{PCl}$ & \pm \pm \pm \\
\hline Zang et a ${ }^{69}$ & Patients with ACS undergoing $\mathrm{PCl}$ & \pm \pm \pm \\
\hline
\end{tabular}

*Represents risk of bias based on: sequence generation of allocation; allocation concealment and blinding. '+' represents low bias risk, '-' high bias risk and ' \pm ' unclear bias risk.

ABCD, Evaluating Additional Benefit of Cilostazol to Dual Antiplatelet Therapy in Patients with Long or Multivessel Coronary Artery Disease underwent Biolimus-Eluting Stent Implantation; ACS, acute coronary syndrome; AMI, acute myocardial infarction; BES, biolimus-eluting stent; BMS, bare metal stent; CIDES, comparison of cilostazol versus clopidogrel after drug-eluting stenting in diabetic patients; CILON-T, Influence of Cilostazol-based Triple Antiplatelet Therapy on Ischaemic Complication After Drug-eluting Stent Implantation; CLEAR, The Cilostazol Administration Before Percutaneous Coronary Intervention for Reduction of Periprocedural Myonecrosis Trial; CREST, Coronary Stent Restenosis in Patients Treated with Cilostazol; DECLARE-LONG II: Triple Antiplatelet Therapy With Dual Antiplatelet Therapy to Reduce Restenosis After Drug-Eluting Stent Implantation in Long Coronary Lesions; DECLARE-DIABETES, A Randomised Comparison of Triple Antiplatelet Therapy with Dual Antiplatelet Therapy After Drug-Eluting Stent Implantation in Diabetic Patients; DECLARE-LONG, Drug-Eluting Stenting Followed by Cilostazol Treatment Reduces Late Restenosis in Patients with Long Coronary Lesions; DES, drug-eluting stent; DM, diabetes mellitus; EES, everolimus-eluting stent; EES-PtCr, everolimus-eluting platinum-chromium alloy stent; LONG-DES, Sirolimus-Eluting Stent Versus Paclitaxel-Eluting Stent for Patients With Long Coronary Artery Disease; OPTIMUS-2, Impact of Cilostazol on Platelet Function Profiles in Patients with Diabetes Mellitus and Coronary Artery Disease on Dual Antiplatelet Therapy; PES, Paclitaxel-eluting stent; SES, Sirolimus-eluting stent; ZES, Zotarolimus-eluting stent; ZES-R, Zotarolimus-eluting Resolute stent.

Other trial expansions as in tables 1 and 2.

\section{DISCUSSION}

In patients undergoing PCI, TAPT using cilostazol results in significant decrease in platelet reactivity and reduced risk of HTPR. TAPT resulted in significantly lower mean PRU, greater platelet inhibition and reduced risk of HTPR in the setting of DAPT with both standard-dose and high-dose clopidogrel. In addition, TAPT was associated with a significant reduction in CV events, including reduction in MACE, driven largely by significant reductions in TLR and TVR. Most importantly, there was a significant lower stent thrombosis with TAPT versus DAPT. Moreover, the reduction of restenosis with TAPT remained even when the analysis was restricted to trials using DES. In addition, there was numerically higher bleeding with TAPT versus DAPT, although this did not reach statistical significant. 


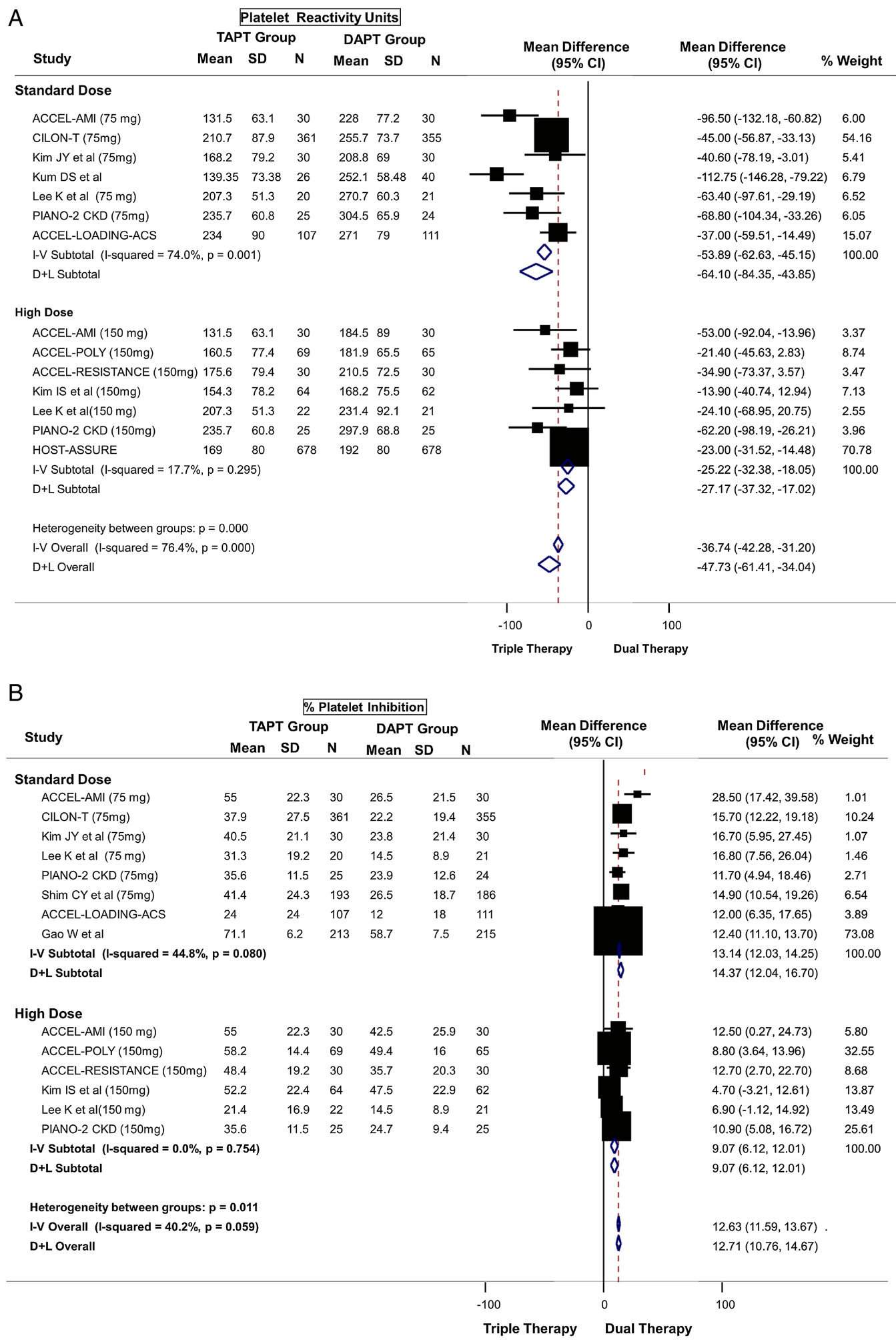

Figure 2 (A) Primary platelet reactivity outcome: difference in platelet reactivity units (PRU) after treatment between triple antiplatelet therapy (TAPT) versus dual antiplatelet therapy (DAPT). (B) Secondary platelet reactivity outcome: difference in percent platelet inhibition after treatment between TAPT versus DAPT. (C) Secondary platelet reactivity outcome: risk of high on-treatment platelet reactivity (HTPR) after treatment between TAPT versus DAPT.

However, there was a significant increase in the risk of drug discontinuation due to adverse effects when compared with DAPT.

\section{Platelet reactivity and outcomes}

Prior studies have shown a relationship between on-treatment platelet reactivity and adverse CV events in 


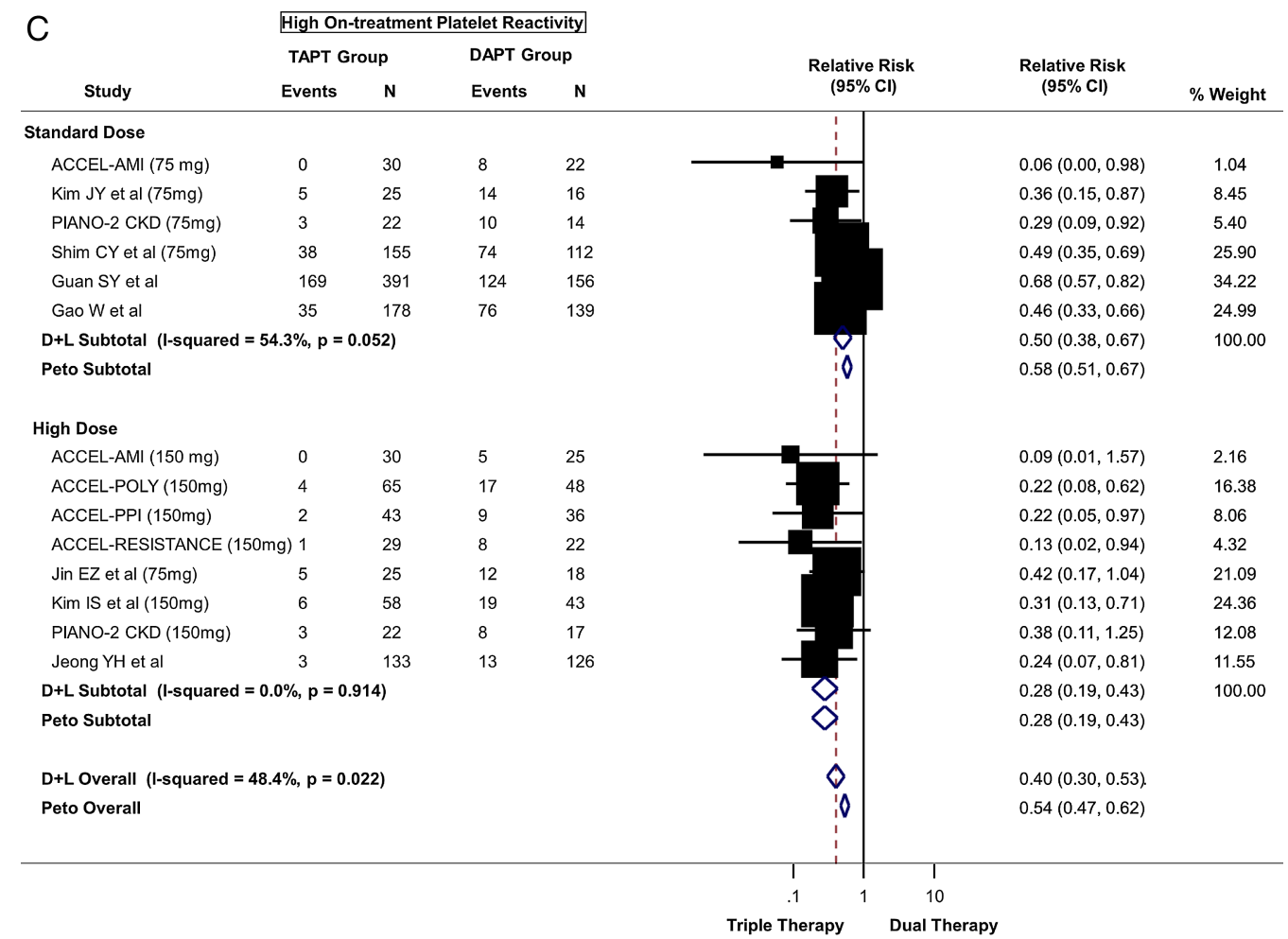

Figure 2 Continued

patients undergoing PCI. In an analysis of individual patient data from six studies with 3059 patients, for every $10 \mathrm{U}$ increase in PRU there was a $4 \%$ increase in primary endpoint rate of death, MI or stent thrombosis (HR 1.04; 95\% CI 1.03 to $1.06 ; \mathrm{p}<0.0001) .{ }^{17} \mathrm{~A}$ recent consensus statement recommended a cut-off of PRU $>235 \mathrm{U}$ as the threshold for identifying patients with HTPR who may be at high risk for ischaemic or thrombotic events following PCI. ${ }^{13}$ Patients with HTPR have been shown to have an increased risk of death $(110 \%$ increase), MI (104\% increase) and stent thrombosis (211\% increase). ${ }^{1718}$

Although platelet reactivity is a surrogate marker, given the wide interindividual variability in clopidogrelinduced platelet inhibition, ${ }^{1-3}$ various strategies have been tested to improve platelet inhibition. These strategies have utilised higher loading and maintenance doses of clopidogrel, or next-generation P2Y12 inhibitors such as prasugrel and ticagrelor, which are more potent that clopidogrel and have a more uniform antiplatelet effect. Doubling of the clopidogrel dose $(150 \mathrm{mg})$ has been shown to significantly reduce PRU in patients with HTPR. ${ }^{19-21}$ Similarly, data from the next-generation P2Y12 inhibitors such as prasugrel and ticagrelor have shown improved platelet reactivity indices when compared with clopidogrel. ${ }^{22}$ Although the newer agents prasugrel and ticagrelor reduce MACE in randomised trials, these agents increase bleeding in patients with PCI and cost significantly more than generic clopidogrel. ${ }^{23} 24$

Cilostazol, a phosphodiesterase III inhibitor, exhibits antiplatelet effects by increasing cAMP within platelets, and is available as a generic drug. Our results show a significant benefit of TAPT with cilostazol in improving platelet reactivity indices in patients undergoing PCI, with lower PRU, greater platelet inhibition and a significant reduction in the risk of HTPR regardless of comparison with either standard-dose or high-dose clopidogrel. In addition, these results were seen even in comparison with DAPT using high-dose clopidogrel. Given that generic clopidogrel is now available, many clinicians opt to prescribe high-dose clopidogrel to address HTPR in patients who cannot afford newer antiplatelet agents. The results of the present study show that TAPT with cilostazol is superior even to DAPT with high-dose clopidogrel. Despite these promising results, a number of limitations must be acknowledged. Although platelet reactivity is a risk factor/surrogate marker for adverse CV events, clinical studies have not yet demonstrated that a pharmacological treatment strategy based on platelet reactivity improves outcomes. ${ }^{20} 25$ In the ARCTIC trial of 2440 patients randomised to platelet-function monitoring and drug adjustment group versus conventional strategy of no monitoring and drug adjustment, there were no differences in composite of death, MI, stent thrombosis, stroke, or urgent revascularisation at 1 year between the two groups, calling into question the utility of adjusting therapies based on platelet function monitoring. ${ }^{25}$

However, because cilostazol inhibits both platelet activation and smooth muscle proliferation, it has the potential to target two dreaded complications of PCI-stent thrombosis and restenosis. TAPT may reduce MACE by two or more cellular mechanisms. ${ }^{8-11}$ Our study shows 


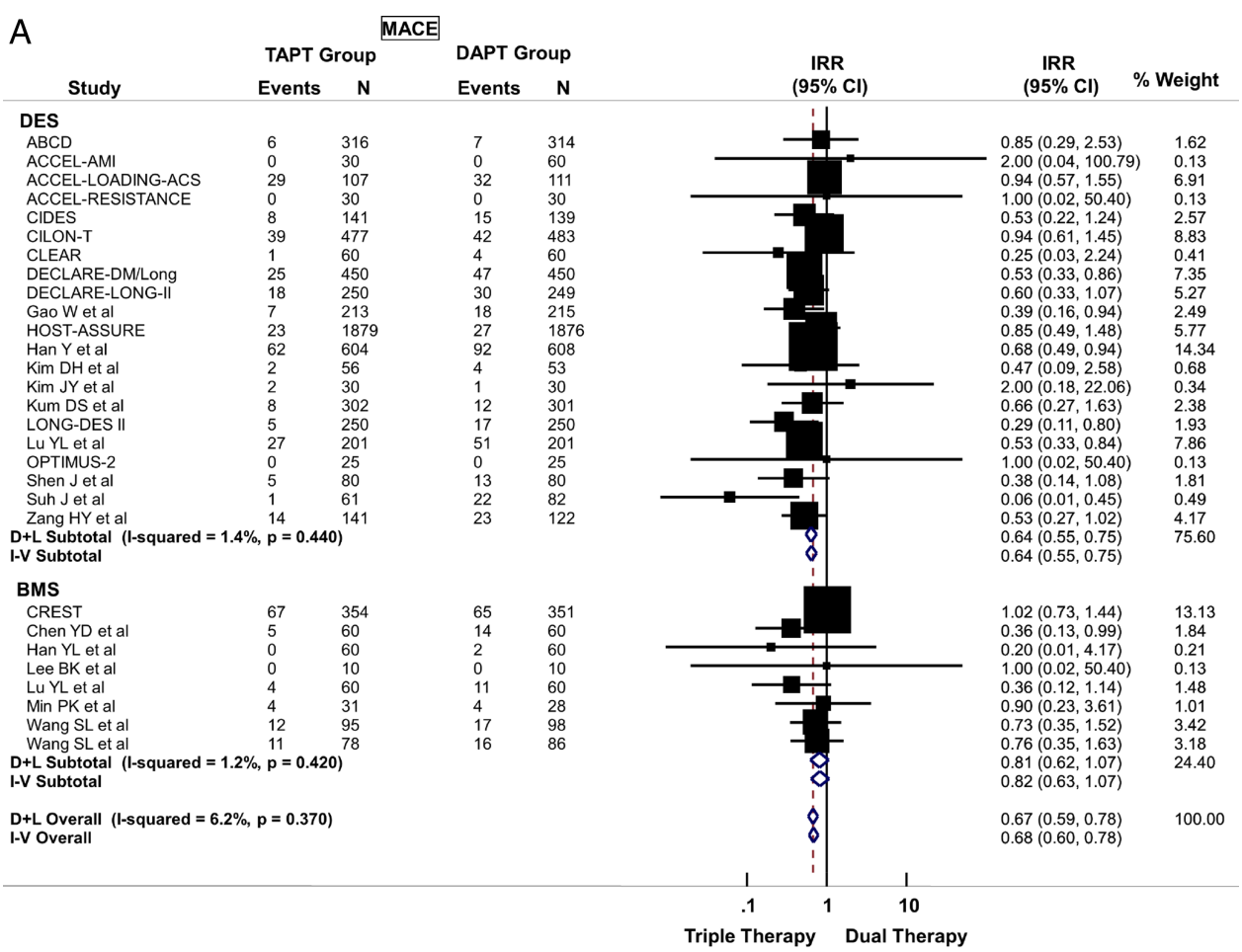

significant reduction in both stent thrombosis and restenosis using TAPT with cilostazol, even in patients treated with DES. This is a potential advantage for this agent, as no antiplatelet agent, including prasugrel or ticagrelor, has been shown to have any antirestenosis property. 

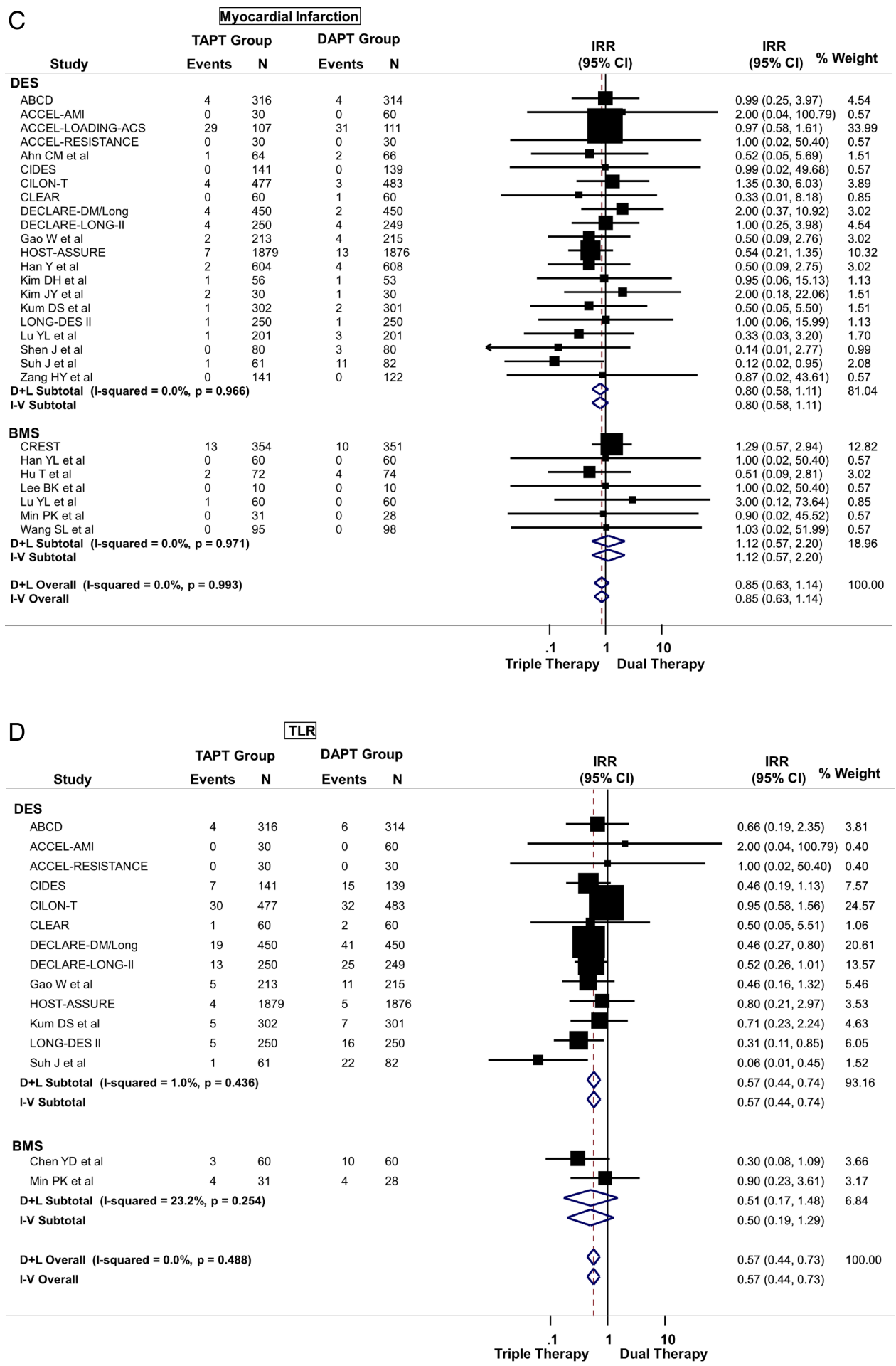

Figure 3 Continued

Therefore, a strategy of using TAPT with cilostazol has several advantages: (1) it improves the surrogate outcome of platelet reactivity relative to DAPT, including high-dose clopidogrel; (2) the antismooth muscle proliferative properties of cilostazol may make it an excellent agent to prevent restenosis resulting in reduced TVR even in patients treated with a DES; (3) the improvement in platelet reactivity indices translate into significant reduction in stent thrombosis and (4) the medication is available generically and is therefore less expensive than newer antiplatelet therapy. Thus, when used following PCI, TAPT with cilostazol has the potential to be a cost-effective therapy to improve clinical outcomes by reducing thrombotic events and restenosis. The results of this study 


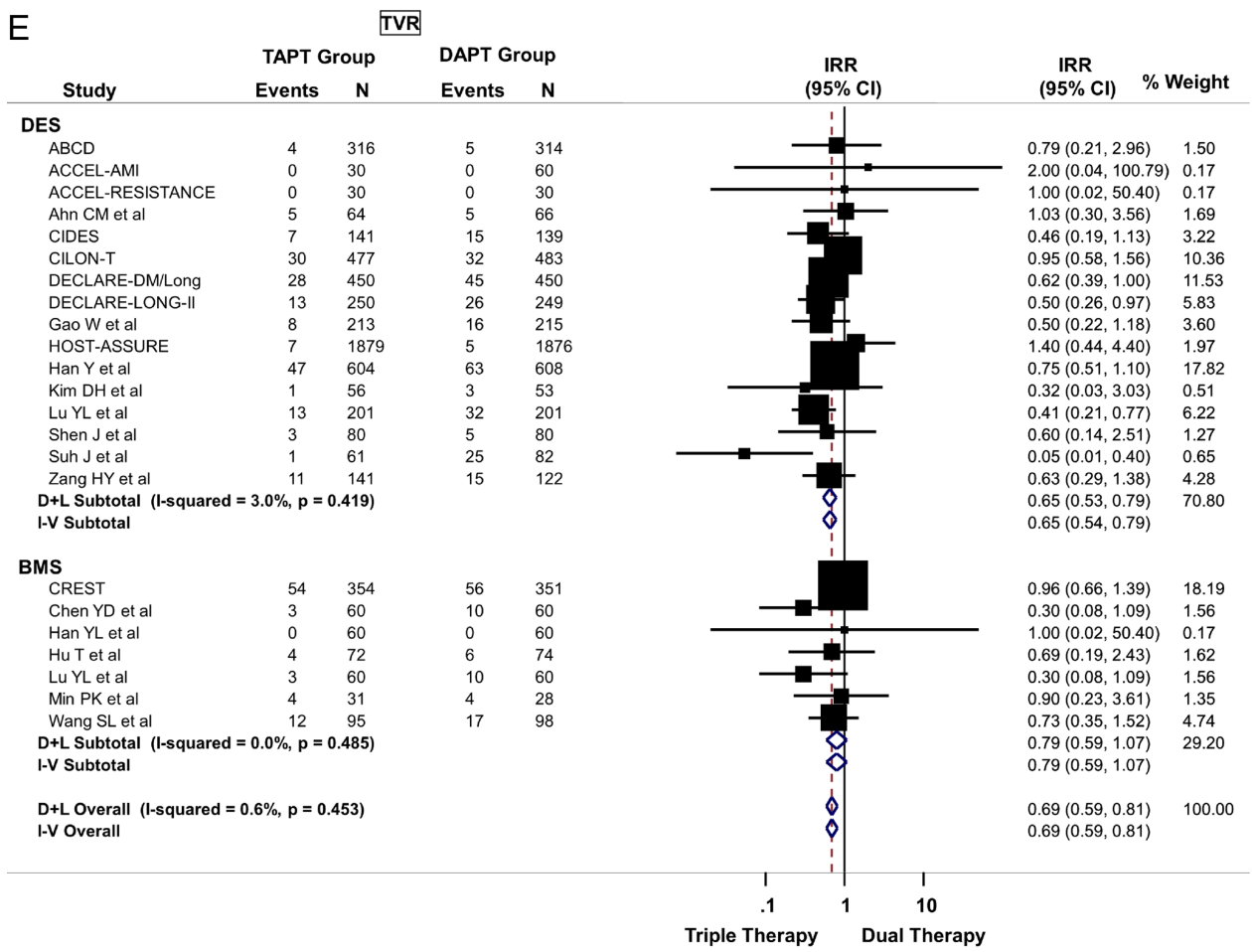

$\mathrm{F}$

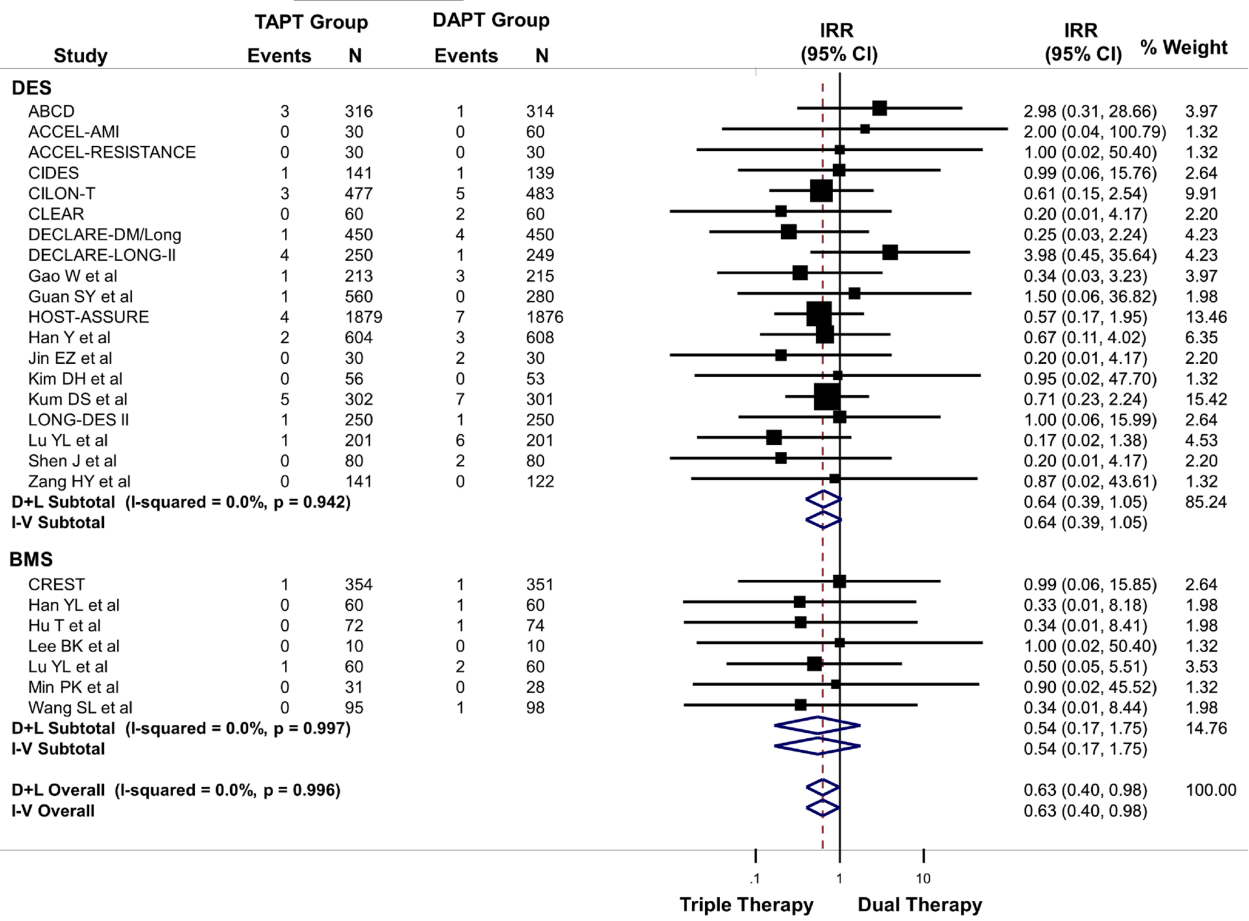

Figure 3 Continued

therefore call for a randomised trial comparing a strategy of TAPT with DAPT using newer antiplatelet agents.

Our results differ from the studies of Jang $e t a l^{26}$ and Sakurai $e t a l^{27}$ in that these studies did not evaluate platelet reactivity outcomes and had far fewer trials than the current analysis. In our analysis, TAPT was associated with significant increase in drug discontinuation. The most commonly listed causes for drug discontinuation were headache, skin rash and palpitations/tachycardia. Sakurai $e t a l^{27}$ similarly found a significant increase in rash and gastrointestinal side effects with TAPT. 

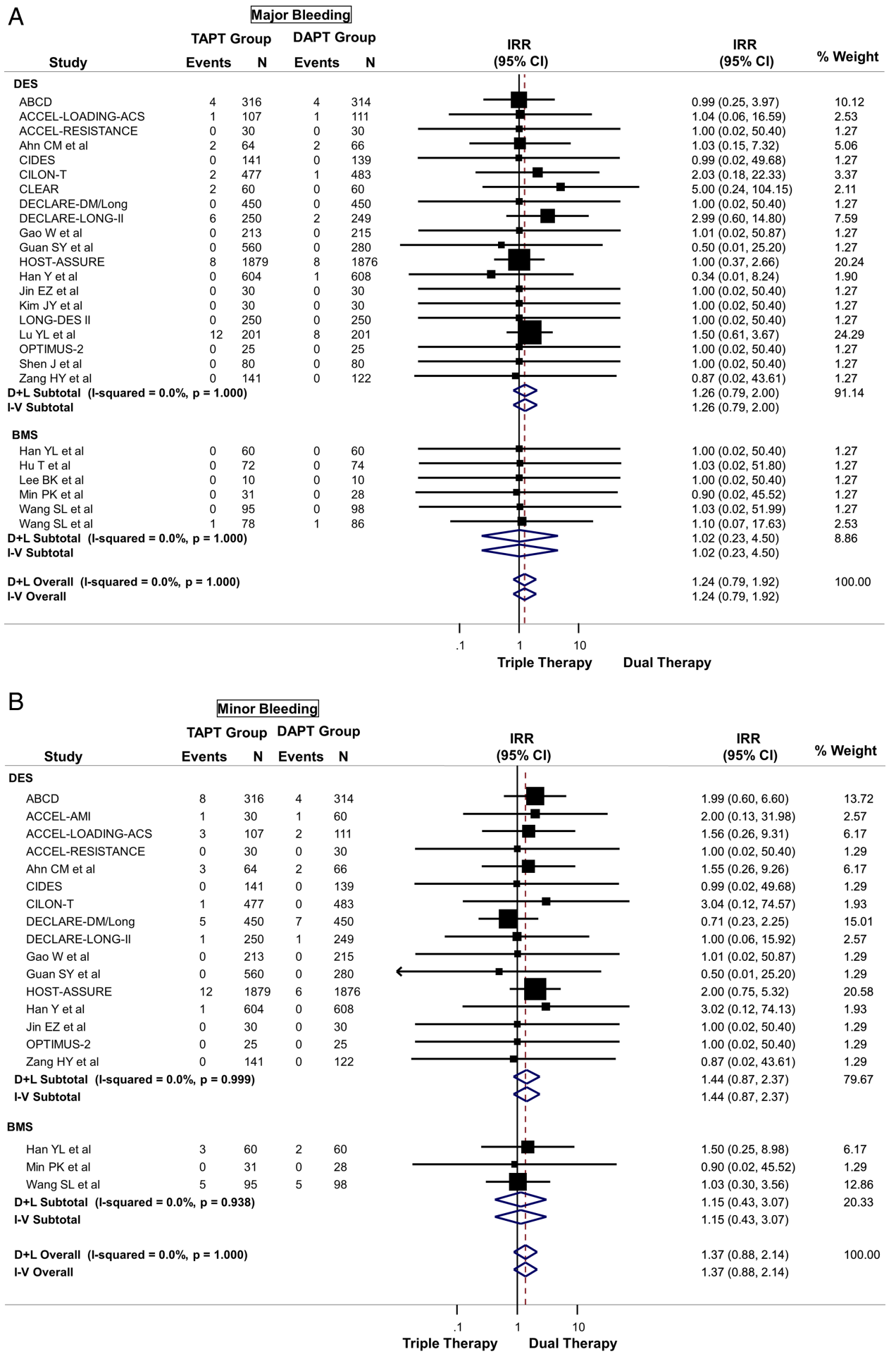

Figure 4 (A) Safety outcome: risk of major bleeding between triple antiplatelet therapy (TAPT) versus dual antiplatelet therapy (DAPT). (B) Safety outcome: risk of minor bleeding between TAPT versus DAPT. (C) Safety outcome: risk of any bleeding between TAPT versus DAPT. (D) Safety outcome: risk of drug discontinuation due to adverse effects between TAPT versus DAPT.

\section{Study limitations}

As in other meta-analyses without individual patient data, we were unable to adjust for dosages of medication used or with compliance with assigned therapies. Given heterogeneity in the study protocols, clinically relevant differences could have been missed and are best assessed in a meta-analysis of individual patient data. Stroke would have been interesting to examine, as there is some evidence that cilostazol reduces stroke. ${ }^{28}$ All of the trials did not report all of the outcomes. The 

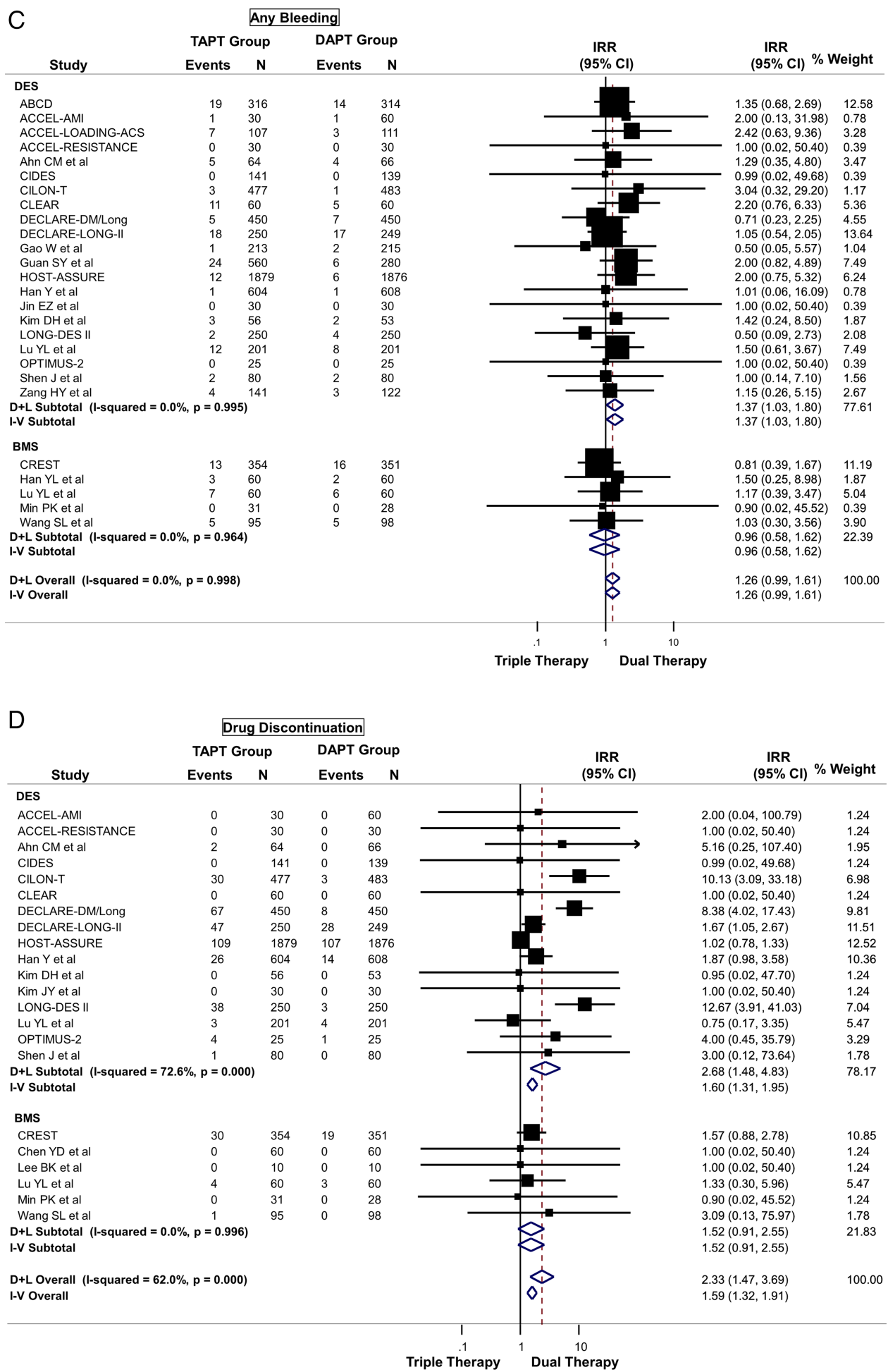

Figure 4 Continued

subgroup analyses might suffer from multiple testing. In addition, the results need to be confirmed in an ethnically diverse population, as most of the trials were done in Asian populations. However, the CREST and the OPTIMUS-2 trials, performed mainly in a non-Asian population, showed similar efficacy of cilostazol when compared with controls. The individual trials did not provide sufficient data to stratify analyses by early versus newer generation DES.

\section{Conclusions}

In patients undergoing PCI, TAPT with cilostazol is associated with significantly improved platelet reactivity indices, even when compared with DAPT with high- 
dose clopidogrel, and is associated with significant reduction in $\mathrm{CV}$ events, including reduction in BMS and DES restenosis and stent thrombosis. The dual properties of antiplatelet and antiproliferative action, the availability as a generic medication combined with the above data makes TAPT with aspirin, clopidogrel and cilostazol an attractive and strong competitor for newer antiplatelet regimens and should be evaluated in future trials.

\author{
Author affiliations \\ ${ }^{1}$ New York University School of Medicine, New York, New York, USA \\ ${ }^{2}$ Saint Luke's Mid America Heart Institute, Kansas City, Missouri \\ ${ }^{3}$ Brigham and Women's Hospital, and Harvard Medical School, Boston, \\ Massachusetts, USA \\ ${ }^{4}$ Brigham and Women's Hospital Heart and Vascular Center and Harvard \\ Medical School, Boston, Massachusetts, USA
}

Contributors SB was involved in study concept and design, analysis and interpretation of the data, statistical analysis, and study supervision and also takes responsibility for the integrity of the data and the accuracy of the data analysis. SB and AS were involved in acquisition of the data and drafting of the manuscript. SB, AS, JJD, KC, FF and DLB were involved in critical revision of the manuscript for important intellectual content.

Funding This research received no specific grant from any funding agency in the public, commercial or not-for-profit sectors.

Competing interests DLB: Advisory Board: Elsevier Practice Update Cardiology, Medscape Cardiology, Regado Biosciences; Board of Directors: Boston VA Research Institute, Society of Cardiovascular Patient Care; Chair: American Heart Association Get With The Guidelines Steering Committee; Data Monitoring Committees: Duke Clinical Research Institute, Harvard Clinical Research Institute, Mayo Clinic, Population Health Research Institute; Honoraria: American College of Cardiology (Editor, Clinical Trials, Cardiosource), Belvoir Publications (Editor in Chief, Harvard Heart Letter), Duke Clinical Research Institute (clinical trial steering committees), Harvard Clinical Research Institute (clinical trial steering committee), HMP Communications (Editor in Chief, Journal of Invasive Cardiology), Population Health Research Institute (clinical trial steering committee), Slack Publications (Chief Medical Editor, Cardiology Today's Intervention), WebMD (CME steering committees); Other: Clinical Cardiology (Deputy Editor), Journal of the American College of Cardiology (Section Editor, Pharmacology); Research Grants: Amarin, AstraZeneca, Bristol-Myers Squibb, Eisai, Ethicon, Medtronic, Roche, Sanofi Aventis, The Medicines Company; Unfunded Research: FlowCo, PLx Pharma, Takeda.

Provenance and peer review Not commissioned; externally peer reviewed.

Data sharing statement No additional data are available.

Open Access This is an Open Access article distributed in accordance with the Creative Commons Attribution Non Commercial (CC BY-NC 3.0) license, which permits others to distribute, remix, adapt, build upon this work noncommercially, and license their derivative works on different terms, provided the original work is properly cited and the use is non-commercial. See: http:// creativecommons.org/licenses/by-nc/3.0/

\section{REFERENCES}

1. Gurbel PA, Bliden KP, Hiatt BL, et al. Clopidogrel for coronary stenting: response variability, drug resistance, and the effect of pretreatment platelet reactivity. Circulation 2003;107:2908-13.

2. Angiolillo DJ, Fernandez-Ortiz A, Bernardo E, et al. Variability in individual responsiveness to clopidogrel: clinical implications, management, and future perspectives. J Am Coll Cardiol 2007;49:1505-16.
3. Serebruany VL, Steinhubl SR, Berger PB, et al. Variability in platelet responsiveness to clopidogrel among 544 individuals. J Am Coll Cardiol 2005;45:246-51.

4. Tousoulis D, Briasoulis A, Dhamrait S, et al. Effective platelet inhibition by aspirin and clopidogrel: where are we now? Heart 2009:95:850-8.

5. Aradi D, Komosci A, Vorobcsuk A, et al. Prognostic significance of high on-clopidogrel platelet reactivity after percutaneous coronary intervention: Systematic review and meta-analysis. Am Heart $J$ 2010;160:543-51.

6. Angiolillo DJ, Capranzano P, Goto S, et al. A randomized study assessing the impact of cilostazol on platelet function profiles in patients with diabetes mellitus and coronary artery disease on dual antiplatelet therapy: results of the OPTIMUS-2 study. Eur Heart $J$ 2008;29:2202-11

7. Liu Y, Shakur Y, Yoshitake M, et al. Cilostazol (pletal): a dual inhibitor of cyclic nucleotide phosphodiesterase type 3 and adenosine uptake. Cardiovasc Drug Rev 2001;19:369-86.

8. Fujinaga K, Onoda K, Yamamoto K, et al. Locally applied cilostazol suppresses neointimal hyperplasia by inhibiting tenascin- $C$ synthesis and smooth muscle cell proliferation in free artery grafts. $J$ Thorac Cardiovasc Surg 2004;128:357-63.

9. Tsai C-S, Lin F, Chen Y, et al. Cilostazol attenuates MCP-1 and MMP-9 expression in vivo in LPS-administrated balloon-injured rabbit aorta and in vitro in LPS-treated monocytic THP-1 cells. $J$ Cell Biochem 2007;103:54-66.

10. Min P-K, Jung J, Ko Y, et al. Effect of cilostazol on in-stent neointimal hyperplasia after coronary artery stenting. Circ $J$ 2007;71:1685-90.

11. Lee S-W, Park S, Yun S, et al. Triple antiplatelet therapy reduces ischemic events after drug-eluting stent implantation: Drug-eluting stenting followed by cilostazol treatment reduces adverse serious cardiac events (DECREASE registry). Am Heart J 2010:284-91.

12. Higgins JP, Green S. Assessing risk of bias in included studies. Cochrane handbook for systematic reviews of interventions version. 500 edn. Oxford: The Cochrane Collaboration, 2008.

13. Bonello L, Tantry US, Marcucci R, et al. Consensus and future directions on the definition of high on-treatment platelet reactivity to adenosine diphosphate. J Am Coll Cardiol 2010;56:919-33.

14. Moher D, Cook DJ, Eastwood S, et al. Improving the quality of reports of meta-analyses of randomised controlled trials: the QUOROM statement. Quality of reporting of meta-analyses. Lancet 1999;354:1896-900.

15. Higgins J, Green S. Cochrane handbook for systematic reviews of interventions. The Cochrane Collaboration, 2008. http://www. cochrane-handbook.org (accessed 12 Dec 2013).

16. Higgins JP, Thompson SG, Deeks JJ, et al. Measuring inconsistency in meta-analyses. BMJ 2003;327:557-60.

17. Brar SS, ten Berg J, Marcucci R, et al. Impact of platelet reactivity on clinical outcomes after percutaneous coronary intervention. A collaborative meta-analysis of individual participant data. J Am Coll Cardiol 2011:58:1945-54

18. Gurbel PA, Tantry US. Acceptance of high platelet reactivity as a risk factor: now, what do we do about it? JACC Cardiovasc Interv 2010;3:1008-10.

19. Barker CM, Murray SS, Teirstein PS, et al. Pilot study of the antiplatelet effect of increased clopidogrel maintenance dosing and its relationship to CYP2C19 genotype in patients with high on-treatment reactivity. JACC Cardiovasc Interv 2010;3:1001-7.

20. Price MJ, Berger PB, Teirstein PS, et al. Standard- vs high-dose clopidogrel based on platelet function testing after percutaneous coronary intervention: the GRAVITAS randomized trial. JAMA 2011;305:1097-105.

21. Price MJ, Angiolillo DJ, Teirstein PS, et al. Platelet reactivity and cardiovascular outcomes after percutaneous coronary intervention: a time-dependent analysis of the Gauging Responsiveness with a VerifyNow P2Y12 assay: Impact on Thrombosis and Safety (GRAVITAS) trial. Circulation 2011;124:1132-7.

22. Michelson AD, Frelinger AL III, Braunwald E, et al. Pharmacodynamic assessment of platelet inhibition by prasugrel vs. clopidogrel in the TRITON-TIMI 38 trial. Eur Heart $J$ 2009;30:1753-63.

23. Wiviott SD, Braunwald E, McCabe $\mathrm{CH}$, et al. Prasugrel versus clopidogrel in patients with acute coronary syndromes. N Engl J Med 2007;357:2001-15.

24. Wallentin L, Becker RC, Budaj A, et al. Ticagrelor versus clopidogrel in patients with acute coronary syndromes. $N$ Engl J Med 2009;361:1045-57. 
25. Collet JP, Cuisset T, Range G, et al. Bedside monitoring to adjust antiplatelet therapy for coronary stenting. $N$ Engl J Med 2012;367:2100-9.

26. Jang JS, Jin HY, Seo JS, et al. A meta-analysis of randomized controlled trials appraising the efficacy and safety of cilostazol after coronary artery stent implantation. Cardiology 2012;122:133-43.

27. Sakurai $\mathrm{R}$, Koo BK, Kaneda $\mathrm{H}$, et al. Cilostazol added to aspirin and clopidogrel reduces revascularization without increases in major adverse events in patients with drug-eluting stents: a meta-analysis of randomized controlled trials. Int J Cardiol 2013;167:2250-8.

28. Kumbhani DJ, Bhatt DL. Secondary prevention of stroke: can we do better than aspirin? Lancet Neurol 2010;9:942-3.

29. Jeong Y-H, Hwang J, Kim I, et al. Adding cilostazol to dual antiplatelet therapy achieves greater platelet inhibition than high maintenance dose clopidogrel in patients with acute myocardial infarction: results of the adjunctive cilostazol versus high maintenance dose clopidogrel in patients with AMI (ACCEL-AMI) study. Circ Cardiovasc Interv 2010;3:17-26.

30. Jeong Y-H. ACCEL-LOADING-ACS-Multicenter randomized trial evaluating efficacy of cilostazol on platelet aggregation, Inflammation, and myonecrosis in ACS patients. TCT-AP 2012.

31. Hwang SJ, Jeong YH, Kim IS, et al. Cytochrome 2C19 polymorphism and response to adjunctive cilostazol versus high maintenance-dose clopidogrel in patients undergoing percutaneous coronary intervention. Circ Cardiovasc Interv 2010;3:450-9.

32. Jeong $\mathrm{YH}, \mathrm{Hwang} \mathrm{S}$, Park T, et al. Pharmacodynamic effects of adding cilostazol versus double-dose clopidogrel in patients with acute myocardial infarction during proton pump inhibitor co-administration (ACCEL-PPI). J Am Coll Cardiol 2012;59:A127.

33. Jeong Y-H, Lee SW, Choi BR, et al. Randomized comparison of adjunctive cilostazol versus high maintenance dose clopidogrel in patients with high post-treatment platelet reactivity: results of the ACCEL-RESISTANCE (Adjunctive cilostazol versus high maintenance dose clopidogrel in patients with clopidogrel resistance) randomized study. J Am Coll Cardiol 2009;53:1101-9.

34. Suh J-W, Lee S, Park K, et al. Multicenter randomized trial evaluating the efficacy of cilostazol on ischemic vascular complications after drug-eluting stent implantation for coronary heart disease: results of the CILON-T (influence of CILostazol-based triple antiplatelet therapy ON ischemic complication after drug-eluting stenT implantation) trial. J Am Coll Cardiol 2011;57:280-9.

35. Gao W, Zhang $\mathrm{Q}, \mathrm{Ge} \mathrm{H}$, et al. Efficacy and safety of triple antiplatelet therapy in obese patients undergoing stent implantation. Angiology 2013;64:554-8.

36. Guan SY, Han YL, Li Y, et al. [Effects of intensive antiplatelet therapy for patients with high on-treatment platelet reactivity after coronary stent implantation]. Zhonghua Xin Xue Guan Bing Za Zhi 2012:40:25-9.

37. Park KW, Kang SH, Park JJ, et al. Adjunctive cilostazol versus double-dose clopidogrel after drug-eluting stent implantation: the HOST-ASSURE randomized trial (Harmonizing optimal strategy for treatment of coronary artery stenosis-safety \& effectiveness of drug-eluting stents \& anti-platelet regimen). JACC Cardiovasc Inter 2013;6:932-42.

38. Jeong YH, Park Y, Koh JS, et al. Reappraisal of Pharmacodynamic Effect of Adjunctive Cilostazol and High-dose Clopidogrel in East Asian ACS Patients. TCT-AP. 2014

39. Jin EZ, Yu L, Li X. Loading effect of $200 \mathrm{mg}$ cilostazol on platelet inhibition in patients undergoing percutaneous coronary intervention. Int Heart J 2012;53:1-4.

40. Kim IS, Jeong YH, Park Y, et al. Platelet inhibition by adjunctive cilostazol versus high maintenance-dose clopidogrel in patients with acute myocardial infarction according to cytochrome P450 2C19 genotype. JACC Cardiovasc Interv 2011;4:381-91.

41. Kim J-Y, Lee K, Shin M, et al. Cilostazol could ameliorate platelet responsiveness to clopidogrel in patients undergoing primary percutaneous coronary intervention. Circ J 2007;71:1867-72.

42. Kum DS, Kim M, Paik J, et al. Clinical effects of additional cilostazol administration after drug-eluting stent insertion Korean Circ $J$ 2009;39:21-5.

43. Lee K, Kim J, Yoo B, et al. Cilostazol augments the inhibition of platelet aggregation in clopidogrel low-responders. J Thromb Hemost 2010;8:2577-9.

44. Woo JS, Kim W, Lee S, et al. Platelet reactivity in patients with chronic kidney disease receiving adjunctive cilostazol compared with a high-maintenance dose of clopidogrel: Results of the effect of platelet inhibition according to clopidogrel dose in patients with chronic kidney disease(PIANO-2 CKD) randomized study. Am Heart J 2011;162:1018-25.
45. Shim CY, Yoon S, Park S, et al. The clopidogrel resistance can be attenuated with triple antiplatelet therapy in patients undergoing drug-eluting stent implantation. Int $J$ Cardiol 2009; $134: 351$

46. Youn YJ, Lee JW, Ahn SG, et al. Multicenter randomized trial of 3-month cilostazol use in addition to dual antiplatelet therapy after biolimus-eluting stent implantation for long or multivessel coronary artery disease. Am Heart J 2014;167:241-8 e1.

47. Ahn C, Hong S, Park J, et al. Cilostazol reduces the progression of carotid intima-media thickness without increasing the risk of bleeding in patients with acute coronary syndrome during a 2-year follow-up. Heart Vessels 2011:26:502-10.

48. Chen $Y-d, L u Y$, Jin Z, et al. A prospective randomized antiplatelet trial of cilostazol versus clopidogrel in patients with bare metal stent. Chin Med J 2006;119:360-6.

49. Ahn $\mathrm{Y}$, Jeong $\mathrm{M}$, Jeong J, et al. Randomized comparison of cilostazol vs clopidogrel after drug-eluting stenting in diabetic patients (CIDES Trial). Circ $J$ 2008;72:35-9.

50. Kim BK, Oh SJ, Yoon SJ, et al. A randomized study assessing the effects of pretreatment with cilostazol on periprocedural myonecrosis after percutaneous coronary intervention. Yonsei Med $J$ 2011;52:717-26.

51. Douglas JS Jr, Holmes DR Jr, Kereiakes DJ, et al. Coronary stent restenosis in patients treated with cilostazol. Circulation 2005;112:2826-32.

52. Lee SW, Park SW, Kim YH, et al. Drug-eluting stenting followed by cilostazol treatment reduces late restenosis in patients with diabetes mellitus the DECLARE-DIABETES trial (A randomized comparison of triple antiplatelet therapy with dual antiplatelet therapy after drug-eluting stent implantation in diabetic patients). J Am Coll Cardiol 2008;51:1181-7.

53. Lee SW, Chun KJ, Park SW, et al. Comparison of triple antiplatelet therapy and dual antiplatelet therapy in patients at high risk of restenosis after drug-eluting stent implantation (from the Declare-Diabetes and Long Trials). Am J Cardiol 2010;105:168-73.

54. Lee SW, Park SW, Kim YH, et al. Comparison of triple versus dual antiplatelet therapy after drug-eluting stent implantation (from the DECLARE-Long trial). Am J Cardiol 2007;100:1103-8.

55. Lee SW, Park SW, Kim YH, et al. A randomized, double-blind, multicenter comparison study of triple antiplatelet therapy with dual antiplatelet therapy to reduce restenosis after drug-eluting stent implantation in long coronary lesions: results from the DECLARE-LONG II (Drug-eluting stenting followed by cilostazol treatment reduces late restenosis in patients with long coronary lesions) trial. J Am Coll Cardiol 2011;57:1264-70.

56. Han Y, Li Y, Wang S, et al. Cilostazol in addition to aspirin and clopidogrel improves long-term outcomes after percutaneous coronary intervention in patients with acute coronary syndromes: a randomized, controlled study. Am Heart J 2009;157:733-9.

57. Han $Y$, Su $Q, L i Y$, et al. The effects of post coronary stenting triple antiplatelet therapies on platelet functions. Chin J Intern Med 2006; 45:635-8.

58. $\mathrm{Hu} \mathrm{T}, \mathrm{Ma} \mathrm{H}, \mathrm{Li} \mathrm{H}$, et al. Efficacy of cilostazol in patients with acute coronary syndrome after percutaneous coronary intervention. $A m$ Ther 2013;20:151-3.

59. Kim DH, Kim J, Moon S, et al. Effects of long-term triple anti-platelet therapy with low dose cilostazol after drug-eluting stent implantation. Korean J Med 2008;74:368-75.

60. Lee B-K, Lee SW, Park SW, et al. Effects of triple antiplatelet therapy (aspirin, clopidogrel, and cilostazol) on platelet aggregation and $\mathrm{P}$-selectin expression in patients undergoing coronary artery stent implantation. Am J Cardiol 2007;100:610-14.

61. Kim YH, Park SW, Lee SW, et al. Sirolimus-eluting stent versus paclitaxel-eluting stent for patients with long coronary artery disease. Circulation 2006;114:2148-53.

62. Kelbaek H, Klovgaard L, Helqvist S, et al. Long-term outcome in patients treated with sirolimus-eluting stents in complex coronary artery lesions: 3-year results of the SCANDSTENT (Stenting coronary arteries in non-stress/benestent disease) trial. J Am Coll Cardiol 2008;51:2011-16.

63. Baumgart D, Klauss V, Baer F, et al. One-year results of the SCORPIUS study: a German multicenter investigation on the effectiveness of sirolimus-eluting stents in diabetic patients. J Am Coll Cardiol 2007:50:1627-34.

64. Lu Y-L, Chen Y, Lu S. Effects of antiplatelet therapy in patients with high platelet aggregability after percutaneous coronary intervention. Chin J Cardiovasc Dis 2007:35:793-6.

65. Menichelli M, Parma A, Pucci E, et al. Randomized trial of sirolimus-eluting stent versus bare-metal stent in acute myocardial infarction (SESAMI). J Am Coll Cardiol 2007;49:1924-30. 
66. Suh J, Kim WJ, Seo HS, et al. Clinical outcome of patients with diabetes mellitus and chronic total occlusion after drug-eluting stenting followed by cilostazol treatment. Eurointervention. 2009;5(Supp E)

67. Ardissino D, Cavallini C, Bramucci E, et al. Sirolimus-eluting vs uncoated stents for prevention of restenosis in small coronary arteries: a randomized trial. JAMA 2004;292:2727-34.

68. Menozzi A, Solinas E, Ortolani P, et al. Twenty-four months clinical outcomes of sirolimus-eluting stents for the treatment of small coronary arteries: the long-term SES-SMART clinical study. Eur Heart J 2009;30:2095-101.

69. Moses JW, Leon MB, Popma JJ, et al. Sirolimus-eluting stents versus standard stents in patients with stenosis in a native coronary artery. N Engl J Med 2003;349:1315-23.

70. Lu YL, Chen YZ, Lv SZ, et al. Effect of cilostazol on restenosis in elderly patients with coronary bare-metal stents implantation. Chin $\mathrm{J}$ Geriatr 2006;25:537-78. 